\title{
Long noncoding RNA Bmncr regulates mesenchymal stem cell fate during skeletal aging
}

\author{
Chang-Jun Li, ${ }^{1,2}$ Ye Xiao, ${ }^{1}$ Mi Yang, ${ }^{1,2,3}$ Tian Su, ${ }^{1}$ Xi Sun, ${ }^{1}$ Qi Guo, ${ }^{1}$ Yan Huang,' and Xiang-Hang Luo ${ }^{1,2}$ \\ 'Department of Endocrinology, Endocrinology Research Center, Xiangya Hospital of Central South University, Changsha, Hunan, China. ${ }^{2}$ Key Laboratory of Organ Injury, Aging and Regenerative Medicine of \\ Hunan Province, Hunan, China. ${ }^{3}$ Department of Orthopaedic Surgery, Johns Hopkins University School of Medicine, Baltimore, Maryland, USA.
}

\begin{abstract}
Bone marrow mesenchymal stem cells (BMSCs) exhibit an age-related lineage switch between osteogenic and adipogenic fates, which contributes to bone loss and adiposity. Here we identified a long noncoding RNA, Bmncr, which regulated the fate of BMSCs during aging. Mice depleted of Bmncr (Bmncr-KO) showed decreased bone mass and increased bone marrow adiposity, whereas transgenic overexpression of Bmncr (Bmncr-Tg) alleviated bone loss and bone marrow fat accumulation. Bmncr regulated the osteogenic niche of BMSCs by maintaining extracellular matrix protein fibromodulin (FMOD) and activation of the BMP2 pathway. Bmncr affected local 3D chromatin structure and transcription of Fmod. The absence of Fmod modified the bone phenotype of Bmncr-Tg mice. Further analysis revealed that Bmncr would serve as a scaffold to facilitate the interaction of TAZ and ABL, and thus facilitate the assembly of the TAZ and RUNX2/PPARG transcriptional complex, promoting osteogenesis and inhibiting adipogenesis. Adeno-associated viral-mediated overexpression of Taz in osteoprogenitors alleviated bone loss and marrow fat accumulation in Bmncr-KO mice. Furthermore, restoring BMNCR levels in human BMSCs reversed the age-related switch between osteoblast and adipocyte differentiation. Our findings indicate that Bmncr is a key regulator of the age-related osteogenic niche alteration and cell fate switch of BMSCs.
\end{abstract}

\section{Introduction}

Guided by genetic and molecular mediators, as well as the local microenvironment, bone marrow mesenchymal stem cells (BMSCs) differentiate into various mature cell types, including adipocytes and osteoblasts (1-3). Age-related osteoporosis is characterized by reduced bone formation and the accumulation of fat in the bone marrow compartment. With age, BMSCs are more inclined to undergo differentiation into adipocytes rather than osteoblasts, resulting in an increased number of adipocytes and a decreased number of osteoblasts, causing osteoporosis (4-6); however, this mechanism is not fully understood.

Long noncoding RNAs (lncRNAs), nonprotein coding transcripts longer than 200 nucleotides, have been reported to play key roles in the regulation of various diseases and biological processes, including cellular differentiation, proliferation, apoptosis, gene regulation, and cancer development (7-10). Although the biological functions of most lncRNAs are not wholly comprehended, lncRNAs have been shown to regulate gene expression patterns by helping bind key transcription factors to promoters and through the physical association of transcription factors themselves (11-14). Yet, there is little research on the role of lncRNAs in the age-related lineage fate switch of BMSCs in the skeleton.

In the present study, we identified a lncRNA, Bmncr, that regulates the fate of BMSCs during aging. Mice with Bmncr defi-

Authorship note: CJL, YX, and MY contributed equally to this work. Conflict of interest: The authors have declared that no conflict of interest exists. License: Copyright 2018, American Society for Clinical Investigation. Submitted: December 5, 2017; Accepted: September 11, 2018 Reference information: J Clin Invest. 2018;128(12):5251-5266. https://doi.org/10.1172/JCI99044. ciency exhibited an osteoporosis-like phenotype characterized by reduced bone mass and accumulated bone marrow fat. Genetic overexpression of Bmncr in mice stimulated bone formation and decreased bone marrow adiposity. After analyzing the lineage fate switch mechanisms of the mesenchymal stem cell (MSC), it revealed that Bmncr upregulated extracellular matrix molecular fibromodulin to anchor BMSCs in trabecular-rich metaphysis regions and also activated the BMP2 pathway in BMSCs. Bmncr also served as a scaffold to assemble TAZ and ABL and facilitated the formation of the TAZ and RUNX2/PPARG transcriptional complex, which promoted osteogenesis and repressed adipogenesis. Our study reveals a new mechanism of lineage shift of BMSCs during aging and may represent a therapeutic strategy against age-related bone loss.

\section{Results}

Bmncr expression in BMSCs decreased with aging. To identify dysregulated lncRNAs in BMSCs during aging, we sorted the Sca- $1^{+}$ $\mathrm{CD} 29^{+} \mathrm{CD} 45^{-} \mathrm{CD} 11 \mathrm{~b}^{-}$BMSCs from young and aged mice using FACS (15), and conducted microarray analysis (Figure 1A). A total of 92 differentially expressed lncRNAs with at least 3-fold change were identified. Among these lncRNAs, 83 were decreased and 9 were increased in BMSCs isolated from aged mice. We selected 5 lncRNAs with the following properties: (a) expression is high in BMSCs and dramatically decreases during aging; (b) evolutionary conservation score greater than $70 \%$; (c) expression in human BMSCs is negatively correlated with age; and (d) no splicing or overlap with any coding gene present in the National Center for Biotechnology Information's RefSeq database, University of California Santa Cruz Genome Browser, or Ensembl genome browser annotations. Among them, NONMMUTO02667 has the best evo- 
A
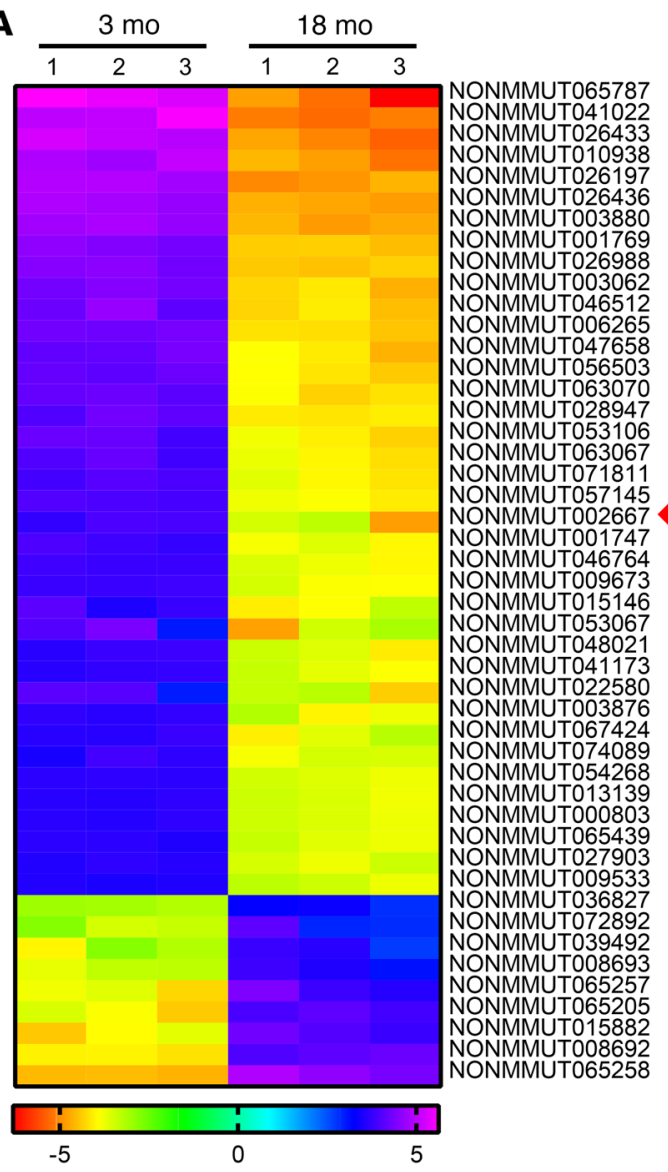

B

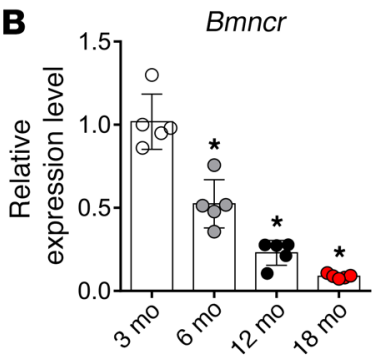

C

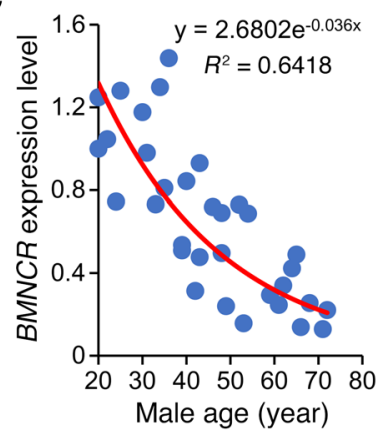

D

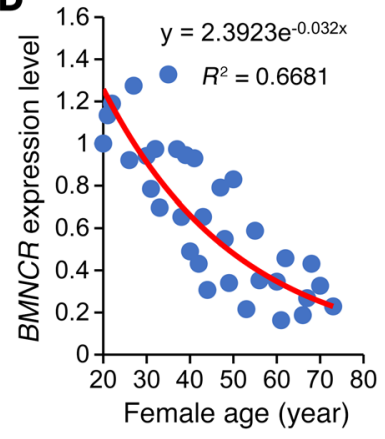

Figure 1. Bmncr expression in BMSCs decreased during aging. (A) Heat map of microarray profiling results of deregulated IncRNAs in BMSCs from young and aged mice (fold change $>3$, false-discovery rate $<0.20$ ). Red arrow indicates the IncRNA (Bmncr) selected for study. (B) qRT-PCR analysis of the levels of Bmncr expression in BMSCs derived from the mice at different ages $(n=5$ per group). (C and $\mathbf{D})$ Age-associated changes of $B M N C R$ levels in human BMSCs from 32 males (C) and 32 females (D). Data are mean \pm SD. ${ }^{*} P<0.01$ (1-way ANOVA).

lutionary conservation. Mammalian sequence conservation scores of NONMMUTO02667 were lower than protein-coding exons but higher than random intergenic regions, in keeping with previous

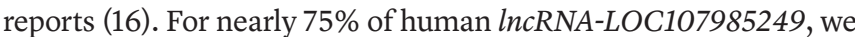
identified orthologous mouse genomic regions of greater than 200 bp, compared with $58 \%$ of random human intergenic regions (Supplemental Figure 1 and 2; supplemental material available online with this article; https://doi.org/10.1172/JCI99044DS1).Therefore, NONMMUTO02667 was selected for further in-depth experiments.

To begin, we measured the NONMMUTO02667 tissue expression pattern in the femur, brain, kidney, lung, liver, heart, and muscle in young mice. A high level of NONMMUT002667 was detected in the femur and white adipocyte tissue (WAT), but not in other tissues (Supplemental Figure 3A). Furthermore, BMSCs and osteoblasts showed a higher level of NONMMUTO02667 expression relative to monocytes and preosteoclasts (Supplemental Figure 3B). We named the candidate Bmncr (bone marrow stem cell-related lncRNA). Bmncr displayed high expression in BMSCs of young mice and significantly decreased during aging (Figure $1 \mathrm{~B})$, as confirmed by quantitative real-time PCR (qRT-PCR).

We isolated human BMSCs (defined as STRO- $1^{+} \mathrm{CD} 146^{+} \mathrm{CD} 45^{-}$) from bone marrow by FACS (17). Similarly, the BMNCR level in human BMSCs was negatively correlated with age (Figure 1, C and
D). These data suggest that Bmncr may play an important role in regulating BMSC functions along with aging in both mice and humans.

Bone marrow microenvironment undergoes age-related changes during aging. Among them, plenty of bone-related hormones (e.g., estrogen), growth factor signaling (e.g., TGF- $\beta$ signaling pathway), and senescence-associated stress (e.g., oxidative stress) changed. To further investigate what is regulating Bmncr expression in BMSCs during aging, we first tested whether estrogen or TGF- $\beta$ pathway would affect Bmncr expression in BMSCs. Bmncr expression level was detected in BMSCs with the administration of $17 \beta$-estradiol and TGF- $\beta 1$. Somewhat unexpectedly, none of the 2 factors affected Bmncr expression in BMSCs (Supplemental Figure 4, A and B). We subsequently measured the effects of oxidative stress on Bmncr expression in BMSCs. Mouse and human BMSCs were treated with buthionine-sulfoximine (BSO), and Bmncr expression was detected. Surprisingly, BSO downregulated Bmncr expression in a dosage-dependent manner (Supplemental Figure 4, C and D). These data indicate that elevated oxidative stress at least partially contributed to the reduced Bmncr expression in BMSCs during aging.

Bmncr knockout mice showed accelerated bone loss and bone marrow fat accumulation. To investigate the role of Bmncr in osteogenic differentiation in adulthood and the aging process, we 
A

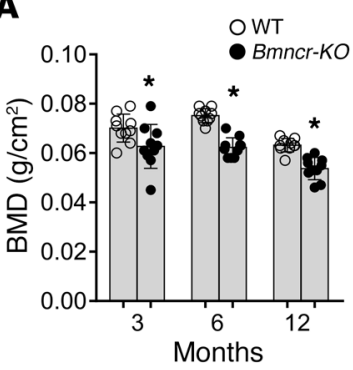

B
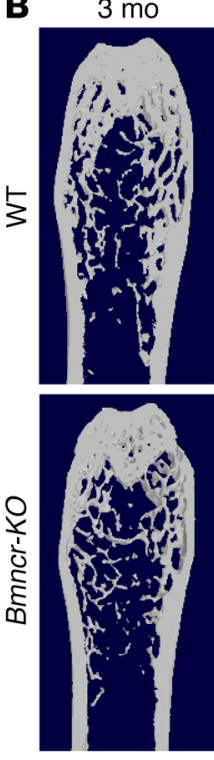

$6 \mathrm{mo}$
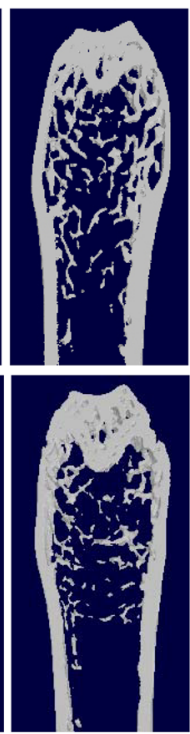

$12 \mathrm{mo}$
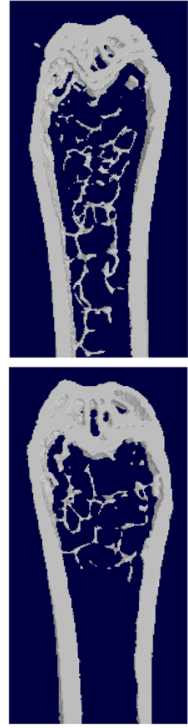

C
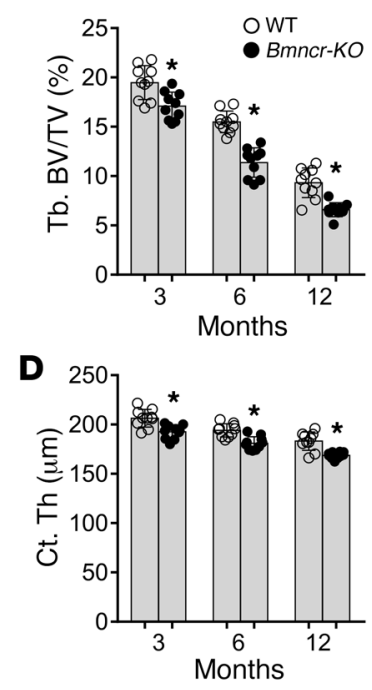
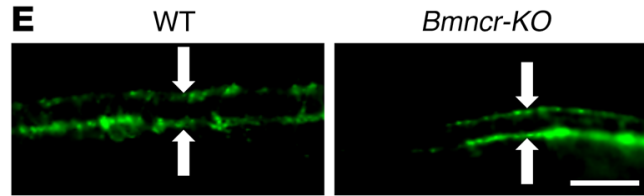

G

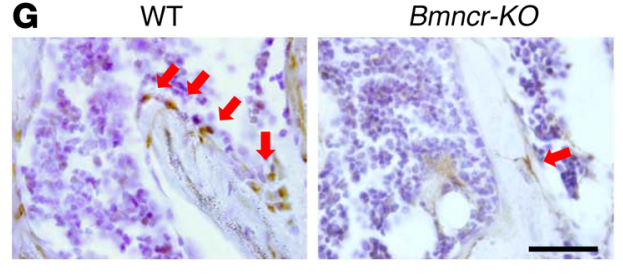

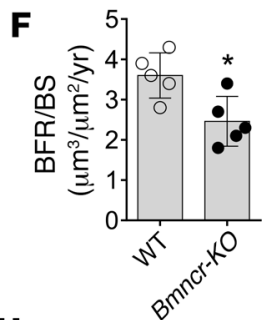

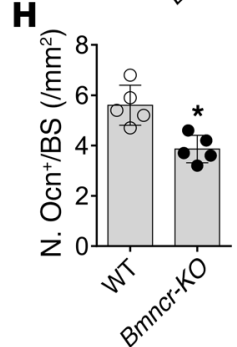

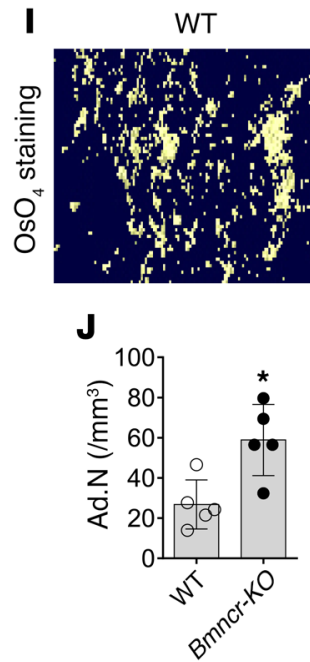

Bmncr-KO

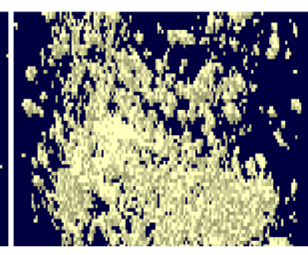

K

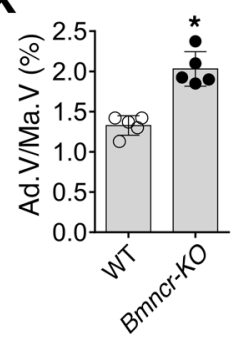

Figure 2. Bmncr-KO mice exhibited lower bone loss and higher bone marrow fat accumulation. (A) BMD in femurs from 3-, 6-, and 12-month-old WT and Bmncr-KO mice were measured by dual-energy x-ray absorptiometry scan. Representative micro-CT images (B) and quantitative micro-CT analysis (C-D) of trabecular bone volume and cortical bone thickness in femurs ( $n=10$ per group). (E-F) Calcein double-labeling-based quantification of bone formation rate per bone surface (BFR/BS) ( $n=5$ per group). Scale bar: $50 \mu \mathrm{M}$. (G-H) Representative images of osteocalcin immunohistochemical staining and quantification of number of osteoblasts in distal femurs. Red arrows represent osteocalcin-positive-staining cells. Scale bar: $100 \mu M$. $n=5$ per group. (I-K) $\mathrm{OsO}_{4}$ staining of decalcified tibiae by micro-CT analysis (I) and quantification of number and volume of adipocytes in femurs $(\mathbf{J}-\mathbf{K})$. $n=5$ per group. Data are mean \pm SD. ${ }^{*} P<0.05$ (Student's $t$ test).

generated Bmncr knockout mice (Bmncr-KO) via gene targeting mediated by the TetraOne technique. qRT-PCR confirmed that the expression level of Bmncr was rarely detected in Bmncr-KO mice relative to wild-type (WT) controls (Supplemental Figure $5 \mathrm{~A})$. We analyzed the bone phenotypes of femurs from young (3-month-old), adult (6-month-old), and aged (12-month-old) mice. The Bmncr-KO mice showed reduced bone mineral density (BMD) in comparison with age-matched WT controls as measured by dual-energy x-ray absorptiometry (DEXA) analysis (Figure 2A). Micro-CT analysis showed that Bmncr-KO mice had lower trabecular bone volume than WT controls. Cortical bone thickness was also reduced in Bmncr-KO mice (Figure 2, B-D). Calcein double-labeling confirmed that Bmncr-KO mice had sig- nificantly lower bone formation rates (BFRs) compared with their WT littermates (Figure 2, E and F).

Immunohistochemical analysis revealed that the number of osteocalcin-positive mature osteoblasts in the bone surface of Bmncr-KO mice was decreased compared with their WT littermates (Figure 2, G and $\mathrm{H}$ ). Moreover, the marrow fat was significantly increased in the bone marrow of Bmncr-KO mice compared with the WT controls as evidenced by a much higher number of adipocytes and fat droplets in decalcified femurs stained with osmium tetroxide $\left(\mathrm{OsO}_{4}\right)$ (Figure 2, I-K).

Histological analysis showed that the number and surface of osteoblasts normalized to the trabecular bone surface were significantly decreased in Bmncr-KO mice, in contrast with age-matched 
A
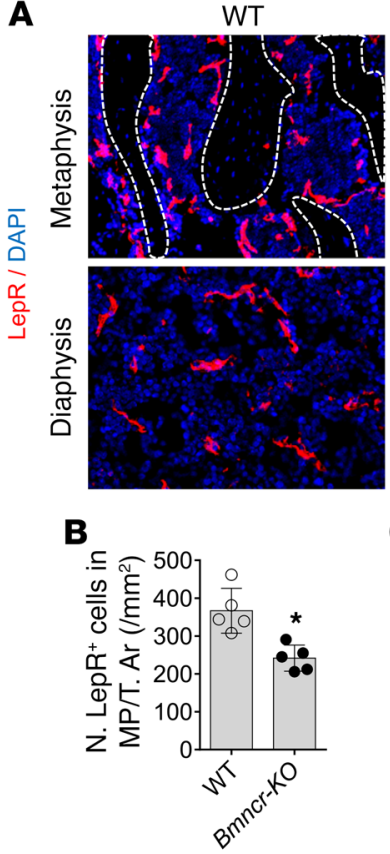

Bmncr-KO

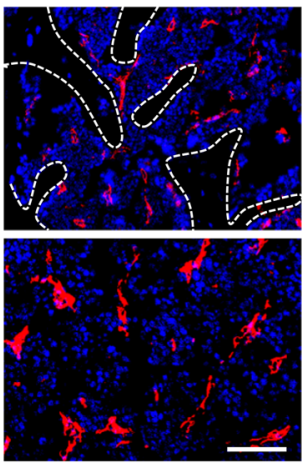

C

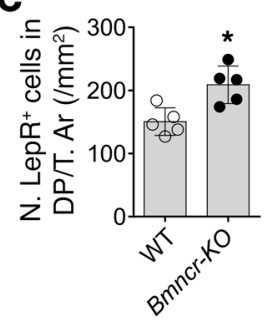

D

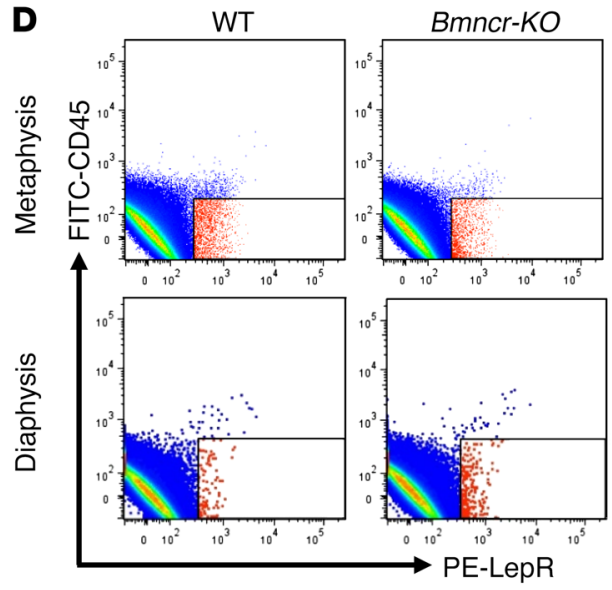

Figure 3. Bmncr-KO mice showed altered LepR ${ }^{+}$BMSC location in bone marrow. (A) Representative immunofluorescence staining images of leptin receptor (LepR, red) in femurs from WT and Bmncr-KO mice. Nuclei, DAPI (blue). Scale bar: $100 \mu \mathrm{M}$. (B-C) Quantitative analysis of the number of LepR $\mathrm{R}^{+}$BMSCs in metaphysis and diaphysis regions of femurs ( $n=5$ per group). (D-E) FACS analysis dot plot (D) and quantitation of LepR+BMSCs (E) ( $n=5$ per group). Data are mean $\pm S D$. ${ }^{*} P<0.05$ (Student's $t$ test).

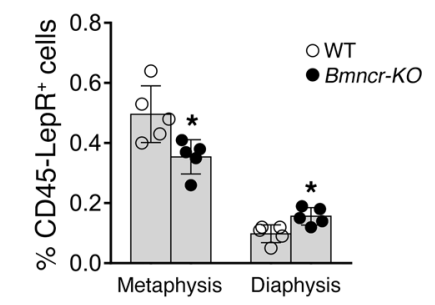

WT controls (Supplemental Figure 5, B and C). However, the number and surface of osteoclasts were not affected in Bmncr-KO mice with normalization of the trabecular bone surface (Supplemental Figure 5, D and E). Together these findings suggest that Bmncr ablation leads to accelerated age-associated bone loss and bone marrow fat accumulation.

Bmncr regulated the osteogenic niche of BMSCs in bone marrow. The lineage decision of stem cells is determined not only by genetic and molecular mediators but also largely by the specific niche of the cell. To investigate the role of Bmncr in regulating the niche of BMSCs in bone marrow, we first localized the BMSCs in vivo. Previous reports show that BMSCs isolated from trabecular-rich metaphysis regions have more proliferation and osteogenic differentiation potential than those in the central bone marrow (18). Immunofluorescence staining analysis revealed that the number of LepR $\mathrm{R}^{+}$cells, multipotent mesenchymal stem/progenitor cells that mainly give rise to osteoblasts and adipocytes in bone (19), significantly decreased in the metaphysis region but increased in the central bone marrow in the femurs of Bmncr-KO mice compared with WT controls (Figure 3, A-C). This observation was further confirmed by flow cytometry analysis (Figure 3, D and E). These results suggest that Bmncr ablation may affect the adherent ability of BMSCs to the bone formation niche. To identify the downstream molecules that mediate Bmncr-induced BMSC location alteration, we FACS-sorted BMSCs from Bmncr-KO mice and WT mice and performed a gene expression profile microarray. A total of 737 differentially expressed genes with at least 1.5 -fold change were identified. Among these genes, an array of extracellular matrix (ECM) protein-encoding genes, including Fmod, Lamb3, Comp, and Col1a, were downregulated in Bmncr-KO BMSCs (Figure 4A). We chose to study the Fmod gene, located 31,066 bp downstream of Bmncr, as it showed the largest differential expression in Bmncr-KO BMSCs. Fmod is a coding gene of fibromodulin, which belongs to the family of small interstitial leucine-rich repeat proteoglycans (SLRPs) (20). Fibromodulin plays an important role in regulating the tendon stem cell niche in the extracellular matrix $(21,22)$. To assess the function of fibromodulin on cell adherence in vivo, Fmod-deficient (Fmod-KO) mice were generated by CRISPR/Cas-mediated genome engineering with Exon 2 as the target $(23,24)$. qPCR analysis showed that Fmod was deleted in Fmod-KO mice (Supplemental Figure 6A). The expression of the Fmod gene in different tissues showed an expression pattern similar to the Bmncr gene, which displayed a high level in the femur and WAT (Supplemental Figure 6B). We first tested whether fibromodulin might modulate BMSC adherent potential to the matrix. BMSCs isolated from Fmod-KO mice and WT mice were subjected to an adhesion assay in matrigel with and without the addition of fibromodulin. We found that Fmod-KO BMSCs showed a reduced ability to adhere to matrigel with and without fibromodulin compared with WT BMSCs. Meanwhile, the number of WT and Fmod-KO BMSCs adherent to matrigel with the addition of fibromodulin was much higher than the number of BMSCs that adhered to matrigel without fibromodulin (Figure 4, B and C). Fmod-KO mice showed significantly decreased LepR $\mathrm{R}^{+}$BMSCs in the trabecular bone surface and increased LepR $\mathrm{R}^{+}$BMSCs in bone marrow as measured by immunofluorescence staining analysis (Supplemental Figure 7, A-C). We also confirmed this result by flow cytometry analysis (Supplemental Figure 7, D and E). These results indicate that Bmncr may regulate the ability of BMSCs to adhere to bone surface matrix through fibromodulin.

We also found that an array of osteogenesis-related genes, including Bmp2, Alpl, Runx2, Sp7, and Bglap, were downregulated in Bmncr-KO BMSCs by gene expression microarray analysis (Figure 4A and Supplemental Figure 8A). Bone morphogenetic protein 2 (BMP2) belongs to the BMP family and is a well-known potent inducer of osteoblast differentiation in MSCs $(25,26)$. To 
A

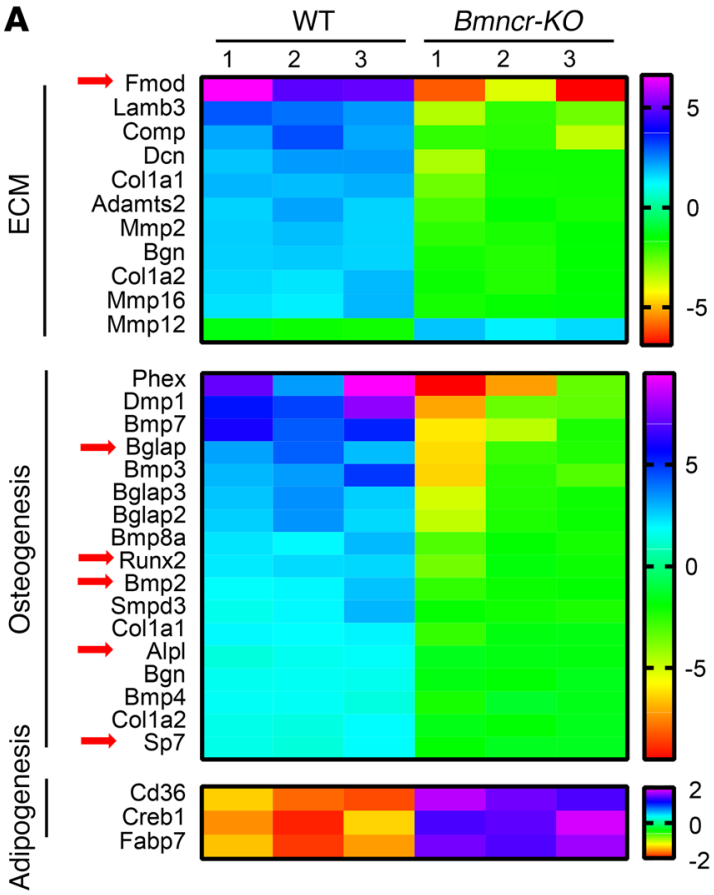

B

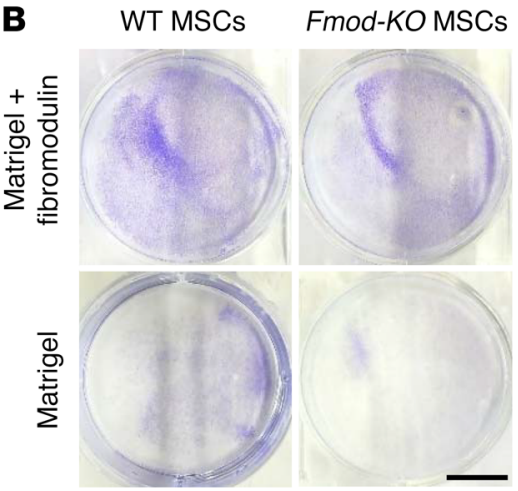

C

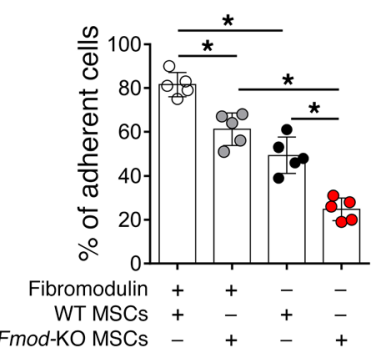

Figure 4. Bmncr regulated osteogenic niche of BMSCs via the FMOD and BMP2 pathway. (A) Heat map of microarray profiling results of deregulated mRNAs in BMSCs from WT and Bmncr-KO mice (fold change $>2$, false-discovery rate $<0.20$ ). (B) Representative image of crystal violet staining of cell adherence assay. Scale bar: $1 \mathrm{~cm}$. (C) Quantitative analysis of the percentage of adherent cells in the indicated groups ( $n=5$ per group). ${ }^{*} P<0.05$ (2-way ANOVA). (D) Western blot analysis of the pSMAD1/5 protein level in BMSCs from WT and Bmncr-KO mice. SMAD1/5 was used as loading control. Data are representative of 3 independent experiments. (E) Schematic model indicating $\mathrm{Bmncr}$ regulates the osteogenic niche of BMSCs through modulating extracellular matrix protein fibromodulin and activation of the BMP2 pathway.
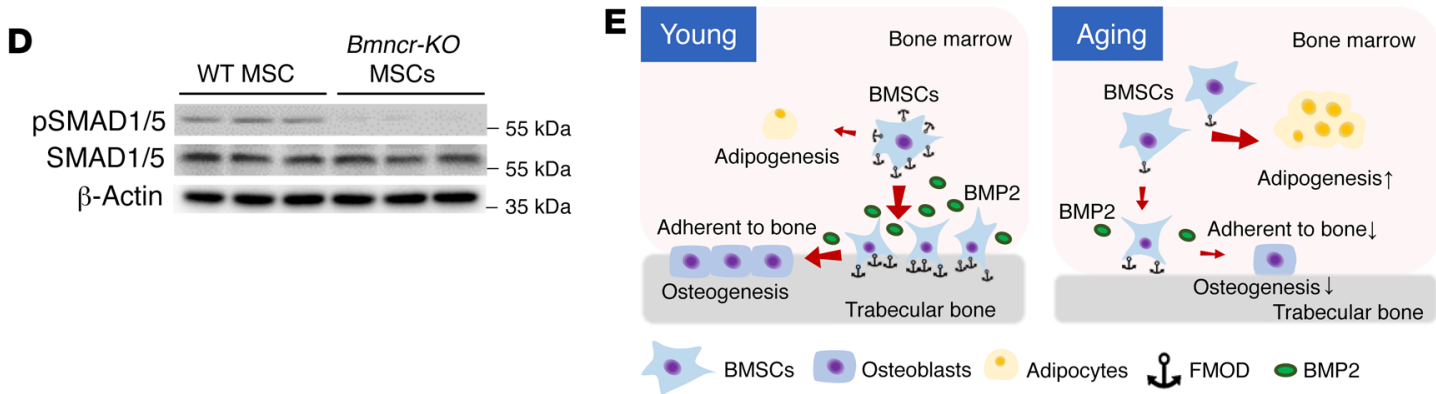

detect the activity of BMP2 signaling in BMSCs, we measured the pSMAD1/5 level by Western blot analysis and found that the level of pSMAD1/5 was significantly decreased in Bmncr-KO BMSCs compared with WT BMSCs (Figure 4D). Moreover, overexpression of Bmncr upregulated the level of pSMAD1/5 in BMSCs (Supplemental Figure 8B). Meanwhile, the adipogenesis-related genes, including Cd36, Creb1, and Fabp7 were upregulated in Bmncr-KO BMSCs (Figure 4A).

These data suggest that Bmncr manipulates the BMSC osteogenic niche through fibromodulin-mediated cell adherence to bone surface and also through the activation of the BMP2 pathway, which may regulate the cell fate shift of BMSCs between osteoblasts and adipocytes (Figure 4E).

Bmncr regulates Fmod transcription and local $3 D$ chromatin structure. We first sought to confirm the effects of Bmncr on Fmod gene transcription. Fmod mRNA and protein levels were decreased in BMSCs transfected with siRNA-Bmncr and increased in BMSCs with overexpression of Bmncr compared with the controls (Figure 5, A and B, Supplemental Figure 9, A and B). In our microarray assay, the Bmp2 expression and BMP2 downstream signal pathway were downregulated in Bmncr-KO MSCs (Figure 4A). As BMP2 has been reported to regulate Fmod gene expression (27), we next detected whether BMP2 mediated the effects of Bmncr on Fmod.
Fmod expression was measured in Bmncr-KO MSCs with the addition of rBMP2. The result showed that restoration of BMP2 did not rescue the decreased expression of Fmod in Bmncr-KO BMSCs, implying that Bmncr may have direct effects on Fmod expression (Supplemental Figure 10).

We then assessed the mechanism underlying the regulation of Bmncr on Fmod transcription. Because Bmncr locates approximately $30 \mathrm{~kb}$ upstream of Fmod, in between the active enhancers and promoter of Fmod (Figure 5, C and D), we hypothesized that Bmncr might regulate the $3 \mathrm{D}$ contacts between the enhancers and the promoter of Fmod. Examination of the Fmod locus using quantitative chromatin conformation capture (3C) assays revealed that 2 nearby upstream enhancers showed reduced contact with the Fmod promoter in the Bmncr knockdown group after normalization of a control region on the Fmod intron (Figure 5E). In contrast, the 2 far-upstream enhancers showed no difference in contact with the Fmod promoter (Figure 5E). These data indicate that Bmncr regulates the transcription of Fmod via modulating contacts between the Fmod promoter and enhancers (Figure 5F).

Transgenic overexpression of Bmncr alleviated bone loss and marrow fat accumulation partially through Fmod. We next tested whether overexpression of Bmncr would promote osteoblastic bone formation and suppress bone marrow fat accumulation. Leptin 
A

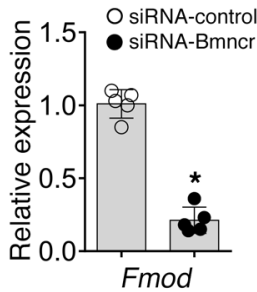

B

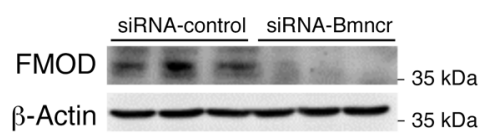

C

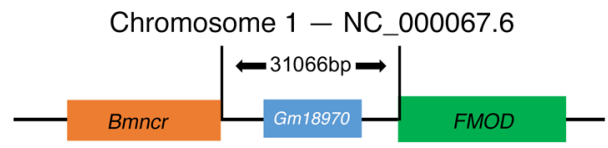

D

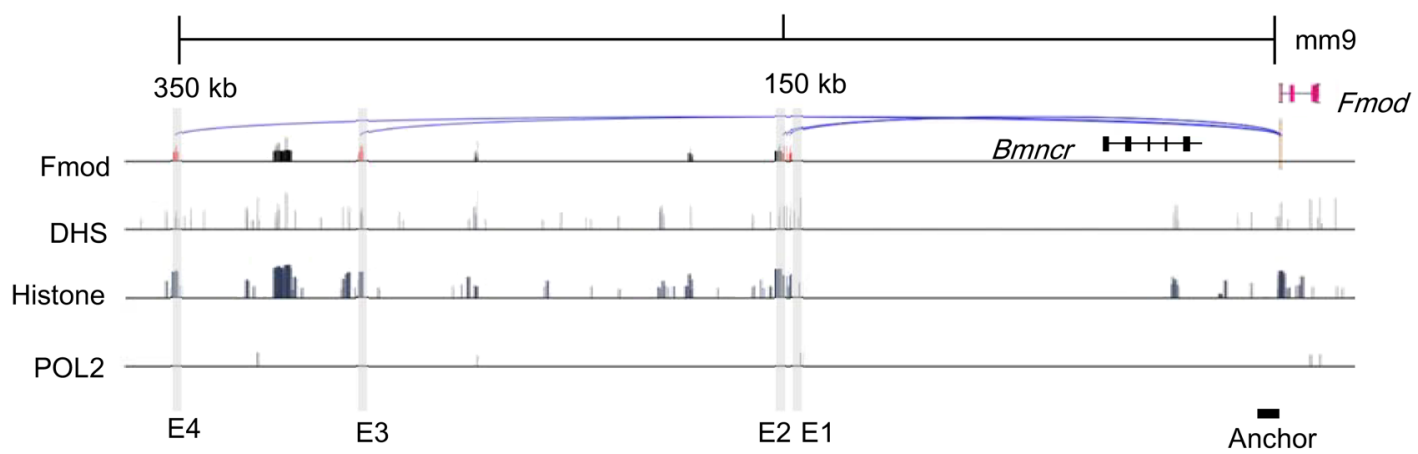

Enhancers associated with Fmod

$\triangle$ Other enhancers
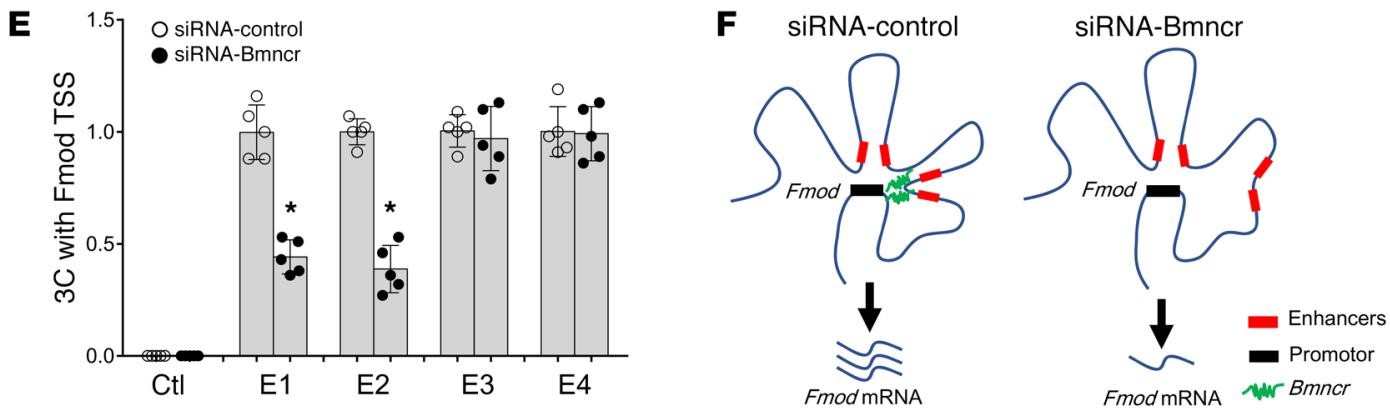

Figure 5. Bmncr regulated Fmod transcription and local 3D chromatin structure. (A) qRT-PCR analysis of the levels of Fmod expression in BMSCs transfected with siRNA-Bmncr or siRNA-control ( $n=5$ per group). Data are mean \pm SD. ${ }^{*} P<0.05$ (Student's $t$ test). (B) Western blot analysis of the FMOD protein level in BMSCs transfected with siRNA-Bmncr or siRNA-control. $\beta$-actin was used as loading control. Data are representative of 3 independent experiments. (C) Schematic of the location of Bmncr and Fmod locus. (D) Schematic of the enhancers and promoter of Fmod locus. (E) $3 C$ analysis revealed that knockdown of Bmncr resulted in reduced contacts between the Fmod promoter (anchor) and 2 enhancers (E1 and E2). Interaction signals were normalized to a control region on the Fmod intron. CTL represents a negative control region that does not harbor interactions with the Fmod promoter ( $n=5$ per group). Data are mean $\pm \mathrm{SD}$. ${ }^{*} P<0.05$ (Student's $t$ test). (F) Schematic model indicating Bmncr knockdown resulted in impaired $3 \mathrm{D}$ contacts between the Fmod promoter and its adjacent enhancer cluster, causing reduced Fmod transcriptional activity.

receptor (LepR) is an excellent marker of multipotent mesenchymal stem/progenitor cells in bone marrow $(19,28)$. To validate LepR as a marker of BMSCs that can differentiate into osteoblasts and adipocytes, we analyzed the LepR-labeled cell lineage using LepR-Cre mice. We consistently found that the LepR-Cre system labels a subpopulation of mesenchymal stromal cells that can differentiate into osteocalcin-positive osteoblasts and perilipin-positive adipocytes, indicating that LepR-expressing cells represent a population of progenitors of osteoblasts and adipocytes (Supplemental Figure 11). Transgenic mice with overexpression of Bmncr in LepR-expressing BMSCs were constructed $\left(\right.$ Bmncr- $\left._{\text {LepR }}^{T_{g}}\right)$. qRTPCR analysis revealed a 6-fold increase in Bmncr expression in BMSCs of Bmncr- $_{\text {LepR }}{ }^{T g}$ mice as compared with that in controls (Supplemental Figure 12A). Bmncr- ${ }_{\text {LepR }}^{{ }^{T g}}$ mice showed increased BMD, higher trabecular bone volume, and increased cortical bone thick- ness in the femurs compared with WT controls (Figure 6, A-C and Supplemental Figure 12, B and C). The femur BFR measured by calcein double-labeling in Bmncr- $^{-}{ }_{\text {LepR }}{ }^{T_{g}}$ mice was also increased dramatically compared with their WT littermates (Figure 6, D and E).

Bmncr- $_{\text {LepR }}{ }^{\text {Ig }}$ mice had a pronouncedly increased number of osteocalcin-positive mature osteoblasts in the trabecular bone surface compared with their WT littermates, as detected by immunohistochemical staining (Figure 6, F and G). Moreover, the number and volume of adipocytes were significantly decreased in bone marrow of $\mathrm{Bmncr-}_{\text {LepR }}{ }^{{ }_{g}}$ mice relative to the WT controls, indicating a positive effect on osteoblastic bone formation but a negative effect on bone marrow fat accumulation of Bmncr (Figure 6, H-J). As expected, a histomorphometric analysis revealed that the number and surface of osteoblasts standardized to the trabecular bone surface were increased in $\mathrm{Bmncr}^{-}{ }_{\mathrm{LepR}}{ }_{\mathrm{Tg}}^{\mathrm{Tg}}$ mice (Supplemental Figure 
A
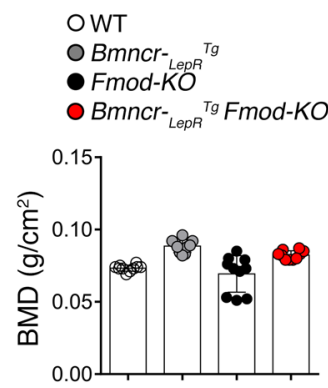

B
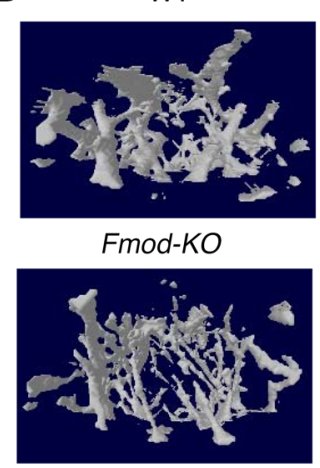

D

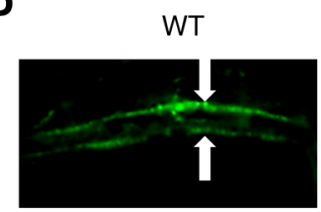

$\mathbf{F}$

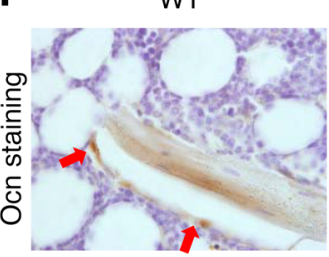

H

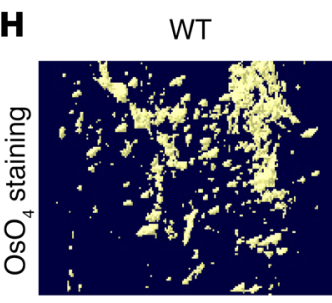

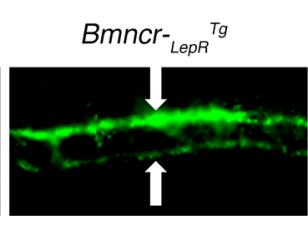

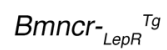

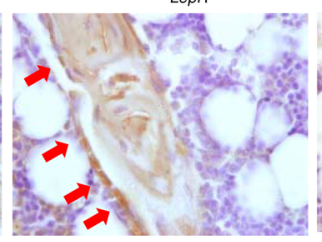

Bmncr- ${ }_{\text {LepR }}{ }^{\top g}$

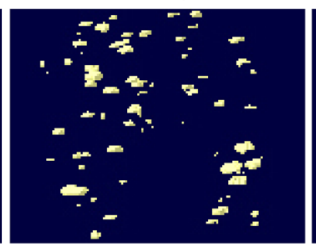

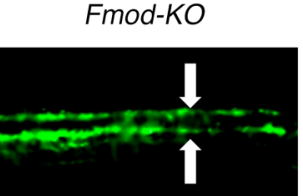

Fmod-KO

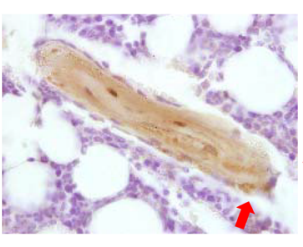

Fmod-KO

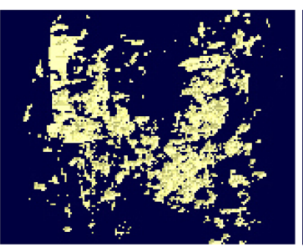

Bmncr- ${ }_{\text {LepR }}^{T g}$ Fmod-KO

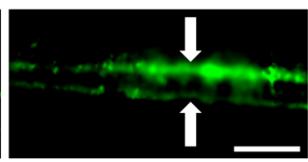

${\text { Bmncr- }{ }_{\text {LepR }}{ }^{\text {Tg }} \text { Fmod-KO }}$

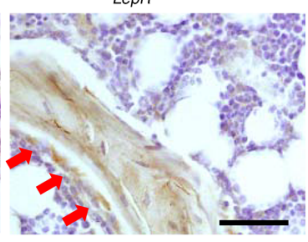

Bmncr- ${ }_{\text {LepR }}{ }^{T_{g}}$ Fmod-KO

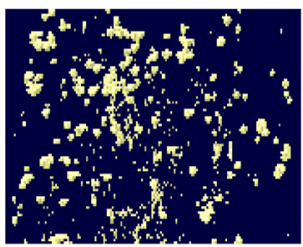

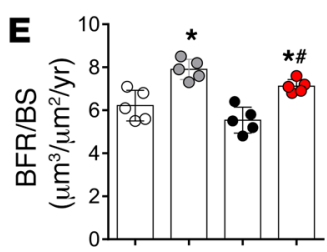
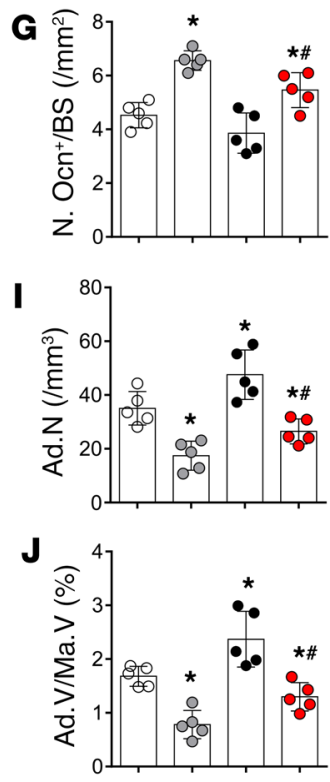

Figure 6. Transgenic overexpression of bmncr alleviated bone loss and marrow fat accumulation partially through Fmod. (A) BMD in femurs from 6-monthold WT and BmnCr- ${ }_{\text {LepR }}{ }^{{ }^{g}}$ mice, Fmod-KO mice, and Bmncr- ${ }_{\text {LepR }}{ }^{{ }^{g}}$ Fmod-KO mice were measured by dual-energy x-ray absorptiometry scan. (B-C) Representative micro-CT images and quantitative micro-CT analysis of trabecular bone mass in femurs ( $n=10$ per group). (D-E) Calcein double-labeling-based quantification of bone formation rate per bone surface (BFR/BS) in femurs $(n=5$ per group). White arrows in $\mathbf{D}$ show the distance between the 2 calcein labeling lines. Scale bar: $50 \mu \mathrm{M}$. Representative images of osteocalcin immunohistochemical staining (F) and quantification of number of osteoblasts (G). Red arrows in $\mathbf{F}$ represent osteocalcin-positive-staining cells. Scale bar: $100 \mu \mathrm{M}$. (H-J) $\mathrm{OsO}_{4}$ staining of decalcified femurs by micro-CT analysis and number and volume of adipocytes in distal femurs. Data are mean \pm SD. $n=5$ per group. ${ }^{*} P<0.05$ versus WT group; ${ }^{\#} P<0.05$ versus Bmncr- ${ }_{\text {LepR }}{ }^{\text {Tg }}$ group (1-Way ANOVA).

13, A and B). However, the number and surface of osteoclasts did not show a significant change in $\mathrm{Bmncr-}_{\text {LepR }}{ }^{\mathrm{Tg}}$ mice (Supplemental Figure 13, C and D). Taken together, these results suggest that overexpression of Bmncr in BMSCs alleviated bone loss and bone marrow fat accumulation.

To further test the role of Fmod in Bmncr effects on bone, we analyzed the bone phenotype of Fmod-KO mice. Although we observed a decreased trend of BMD, trabecular bone mass, cortical bone thickness, BFR, and osteoblast number in Fmod-KO mice compared with WT mice, the changes were not significant (Figure 6, A-G, and Supplemental Figure 12, B and C). However, the adipocyte number and volume were significantly higher in Fmod-KO mice compared with WT mice (Figure 6, $\mathrm{H}^{-} \mathrm{J}$ ). Next, we crossed the Fmod-KO mice with Bmncr-Tg mice to generate Bmncr-Tg Fmod-KO mice to determine whether the absence of Fmod would modify the phenotype of Bmncr-Tg mice. Bmncr-Tg Fmod-KO mice showed a decrease in BMD, trabecular bone mass, cortical bone thickness, and the number of osteoblasts, as well as an increase in adipocyte number and volume compared with those of Bmncr-Tg mice, suggesting that Fmod mediates the effects of Bmncr on bone mass (Figure 6, A-J). However, the absence of Fmod did not modify the phenotype of Bmncr-Tg mice to the level of the WT mice, indicating that in addition to Fmod, Bmncr also regulates bone mass through other signaling pathways. 
A
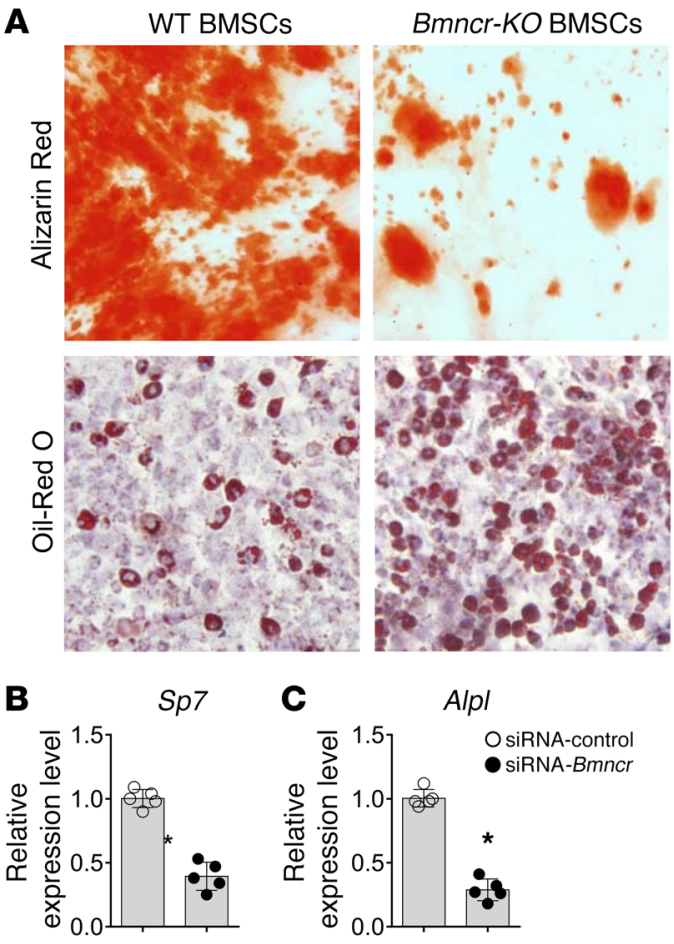

\section{c}

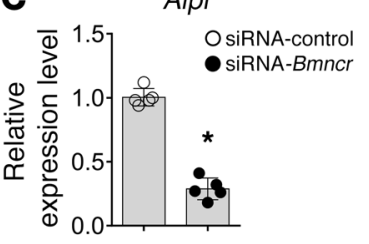

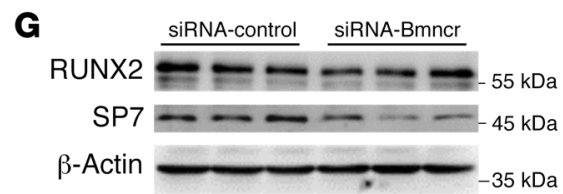

H

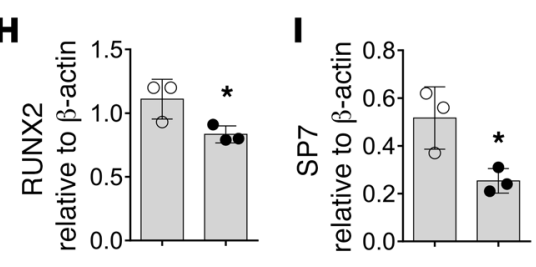

J

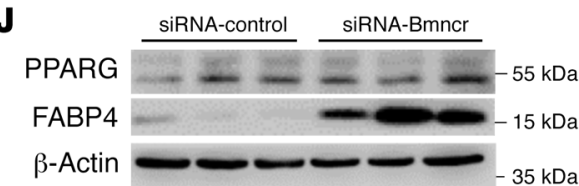

$\mathbf{K}$

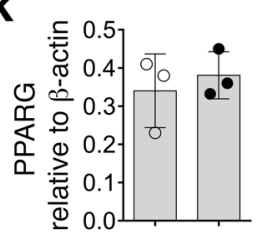

$\mathbf{L}$

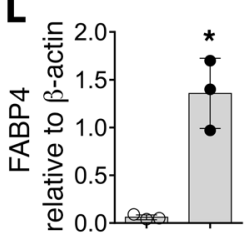

Figure 7. Bmncr promoted osteogenic differentiation and inhibits adipogenic differen-

tiation of mouse BMSCs. (A)

Representative images of Alizarin Red S staining (original magnification $\times 100$ ) and Oil Red 0 staining (original magnification $\times 800$ ) in BMSCs isolated from WT and Bmncr-KO mice with osteogenic induction or adipogenic induction respectively. (B-F) qRT-PCR analysis of the levels of Sp7, Alpl, Bglap, Pparg, and Fabp4 expression in BMSCs transfected with siRNA-Bmncr or siRNA-control. (G-L) Western blot analysis of the RUNX2, SP7, PPARG, and FABP4 protein level. $\beta$-actin was used as loading control. Data are representative of 3 independent experiments. Data are mean $\pm \mathrm{SD}$. ${ }^{*} P<0.05$ (Student's $t$ test).
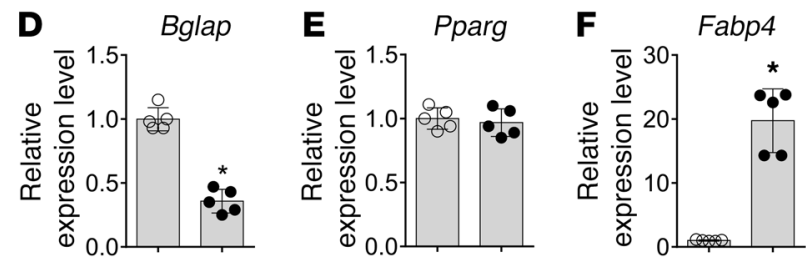

Bmncr regulated lineage fate of BMSCs between osteoblast and adipocyte differentiation. Collectively, our results imply that, in addition to Fmod, Bmncr may have direct effects on cell fate switch of BMSCs between osteogenesis and adipogenesis. To validate the effects of Bmncr on the lineage differentiation of BMSCs, we isolated BMSCs from WT and Bmncr-KO mice and conducted the osteogenic and adipogenic differentiation. Ablation of Bmncr significantly inhibited osteogenic differentiation but facilitated lipid droplet formation of BMSCs (Figure 7A). The mRNA levels of Sp7, Alpl, and Bglap, 3 key markers of osteogenic differentiation, were decreased with Bmncr knockdown (Figure 7, B-D). Western blot analysis consistently revealed that the osteoblast transcription factors RUNX2 and SP7 were decreased in BMSCs with Bmncr knockdown (Figure 7, G-I). In contrast, fatty acid binding protein 4 (Fabp 4), an essential marker of adipocyte differentiation, was increased nearly 20 -fold in BMSCs transfected with siRNABmncr compared with the control group. Pparg mRNA level was not affected by Bmncr deficiency, indicating that Bmncr may regulate PPARG activity rather than transcription (Figure 7, E and F). Western blot analysis confirmed that FABP4, but not PPARG, was increased in siRNA-Bmncr-transfected BMSCs (Figure 7, J-L). These in vitro data suggested that Bmncr has direct effects on the lineage fate of BMSCs.

Bmncr served as a scaffold to assemble $A B L$ and TAZ. To further assess the underlying mechanism of Bmncr regulation of BMSC lineage fate, we conducted mass spectrometry after RNA pull-down with biotinylated Bmncr in BMSCs. Among the identified proteins, we chose TAZ and ABL for further study (Figure 8A). TAZ, a Hippo pathway component, forms a transcriptional complex with RUNX2 that drives osteogenic differentiation of BMSCs, and TAZ coordinately represses adipocyte differentiation in a transcriptional repressor of PPARG-induced gene expression (29). ABL is required for the assembly and activation of the TAZ and RUNX2 master transcription factor complex and the repression of PPARG-mediated adipogenesis (30). We hypothesized that Bmncr might coordinately interact with both TAZ and ABL to regulate osteogenesis and adipogenesis. Purified biotinylated $B m n c r$, but not antisense Bmncr fragments, specifically retrieved TAZ and ABL (Figure $8 \mathrm{~B}$ ). To further validate the interaction between Bmncr and TAZ or ABL, we conducted RNA immunoprecipitation (RIP) of BMSCs. TAZ antibody pulled down significantly more Bmncr than the IgG control as measured by qRT-PCR analysis. In contrast, no differential enrichment of U1 RNA was observed (Figure 8C). Meanwhile, the interaction between ABL and Bmncr was also confirmed by qRT-PCR analysis of ABL antibody-enriched RNA (Figure 8C).

We subsequently looked to map which region of the Bmncr transcript binds with the TAZ or ABL protein. A series of Bmncr deletion mutants was generated and biotinylated for RNA pull-down assay. Deletion mutants that retained nucleotides 1-400 bound TAZ with equal efficiency as full-length Bmncr, indicating the TAZ-binding activity of nucleotides 1-400 of Bmncr. The deletion mutants that 
A ABL-EMDGQPDR

Extracted from: E:IFan_Data20170914IYM-qian.raw \#1510 RT: 14.09

FTMS, HCD $@ 35.00, z=+2$, Mono $\mathrm{m} / \mathrm{z}=474.20334 \mathrm{Da}, \mathrm{MH}+=947.39940 \mathrm{Da}$, Match Tol. $=0.05 \mathrm{Da}$

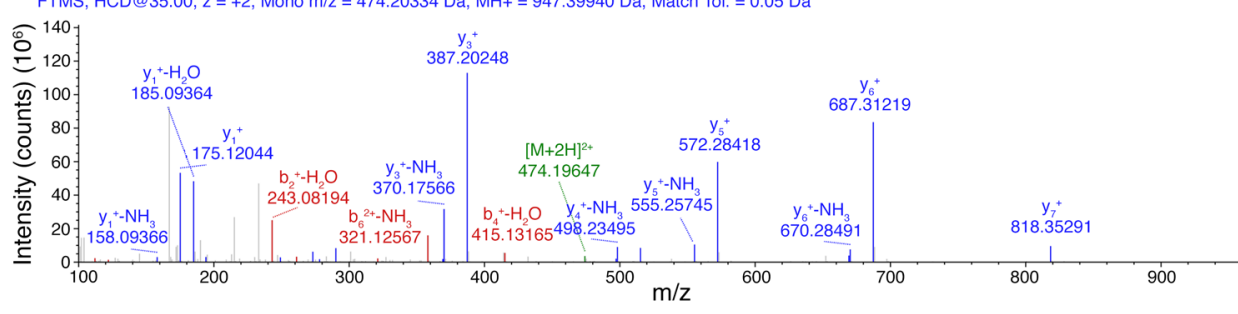

- Pre $+\mathrm{H}$, precursor, precursor- $\mathrm{H}_{2} \mathrm{O}$, precursor- $\mathrm{H}_{2} \mathrm{O}-\mathrm{NH}_{3}$, precursor- $\mathrm{NH}_{3}$, Pre- $\mathrm{H}-\mathrm{y}, \mathrm{y}-\mathrm{H}_{2} \mathrm{O}, \mathrm{y}-\mathrm{NH}$ - b, b- $\mathrm{H}_{2} \mathrm{O}, \mathrm{b}-\mathrm{NH}_{3}$

\section{TAZ-QEAALCR}

Extracted from: E:IFan_Datal20170914\YM-gian.raw \#2030 RT: 16.25

FTMS, HCD@35.00, $z=+2$, Mono m/z $=424.21365 \mathrm{Da}, \mathrm{MH}+=847.42003 \mathrm{Da}$, Match Tol. $=0.05 \mathrm{Da}$

$$
\text { 员 } 400
$$
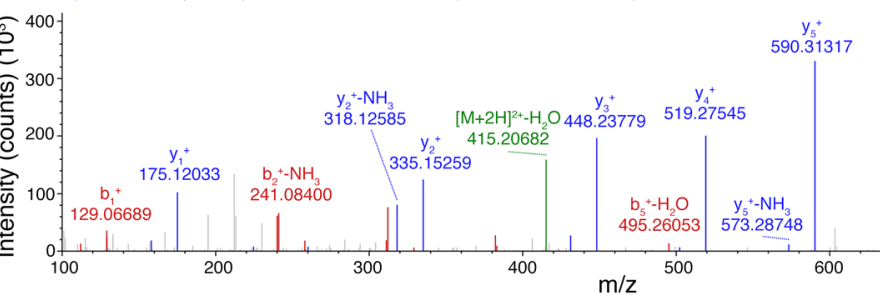

, precursor- $\mathrm{NH}_{3}$, Pre-H

b, b- $\mathrm{H}_{2} \mathrm{O}, \mathrm{b}-\mathrm{NH}_{3}$

D

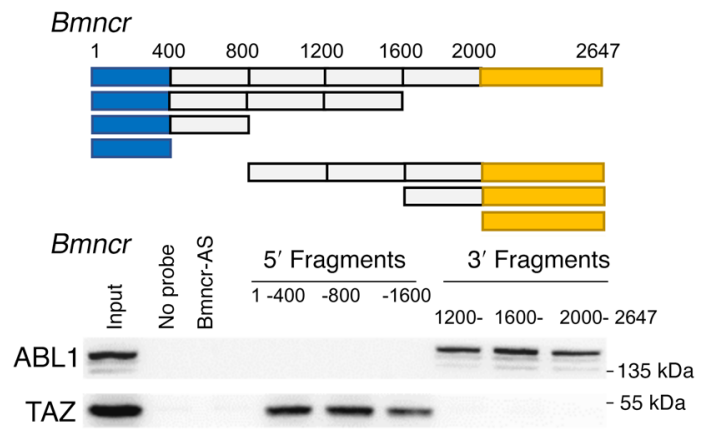

E

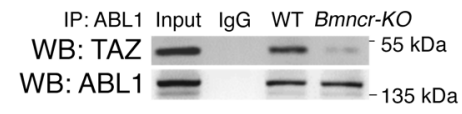

$\mathbf{F}$

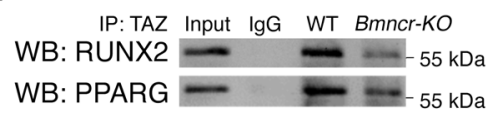

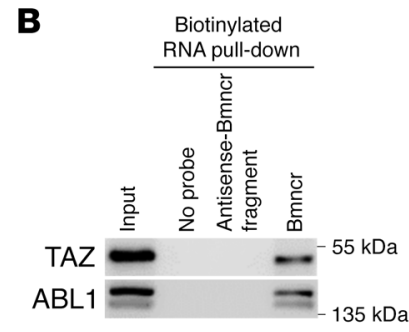

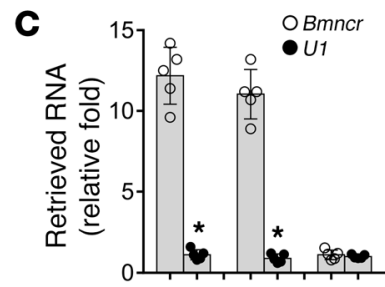

Figure 8. Bmncr served as a scaffold to assemble TAZ and ABL. (A) TAZ and ABL proteins interacting with $B m n c r$ were identified by mass spectrum after RNA pull-down with biotinylated Bmncr in BMSCs. (B) Biotinylated Bmncr retrieves TAZ and ABL as detected by immunoblotting. (C) TAZ and ABL retrieves $B m n c r$ RNA specifically as detected by qRT-PCR. $n=5$ per group. Data are mean \pm SD. ${ }^{*} P<0.05$ (Student's $t$ test). (D) The diagram indicating the first 400 bp (blue boxes) of Bmncr is necessary and sufficient to bind TAZ; the last 647 bp (yellow boxes) is necessary and sufficient to bind ABL. The profiles were established by means of RNA pull-down assay. (E) Immunoprecipitation assays using antibody against ABL, the ABL-associated TAZ was detected by Western blotting with antibody against TAZ. (F) Immunoprecipitation assays using antibody against TAZ, the TAZ-associated RUNX2, and PPARG were detected by Western blotting with antibody against RUNX2 and PPARG. Data are representative of 3 independent experiments.

retained nucleotides 2000-2647 retained the ABL binding ability (Figure 8D). Accordingly, Bmncr is a modular bifunctional RNA that has distinct binding domains for TAZ and ABL.

To assess the direct binding of TAZ and ABL in BMSCs, cell lysates were subjected to immunoprecipitation by an anti-ABL antibody, which was followed by Western blotting using an antiTAZ antibody. The result showed strong binding of TAZ in ABL immunoprecipitates, but not in IgG controls, in the BMSCs of WT mice. Intriguingly, the intensity of the binding was much weaker in BMSCs isolated from Bmncr-KO mice (Figure $8 \mathrm{E}$ ). We then detected whether the impaired binding of TAZ and ABL would affect the transcriptional complex formation of TAZ and RUNX2 or TAZ and PPARG. Immunoprecipitation analysis showed robust binding between TAZ and RUNX2 in BMSCs of WT mice, whereas the binding was much weaker in Bmncr-KO BMSCs relative to the WT control (Figure 8F). Moreover, the RUNX2 transcription activities on its target genes including Sp7, Bglap, Alpl, and Col1a2 were reduced in Bmncr-KO BMSCs (Supplemental Figure 14). We additionally observed strong binding between TAZ and PPARG in WT BMSCs. The binding density of TAZ and PPARG decreased dramatically after Bmncr ablation (Figure 8F). In contrast, BMSCs with overexpression of Bmncr showed enhanced binding of TAZ and ABL and facilitated the complex formation of TAZ-RUNX2 and TAZ-PPARG (Supplemental Figure 15).

These results suggest that Bmncr may serve as a scaffold to facilitate the interaction of TAZ and ABL, as well as the assembly of TAZ-RUNX2 and TAZ-PPARG transcriptional complexes, which subsequently promote osteogenesis and inhibit adipogenesis (Supplemental Figure 16).

Overexpression of Taz partially rescued the premature bone aging of Bmncr-KO mice. Since Bmncr mediated TAZ activities, we next tested whether overexpression of Taz would rescue the bone phenotype 
OWT + rAAV9-Sp7-GFP

A OBmncr-KO + rAAV9-Sp7-GFP

A Bmncr-KO + rAAV9-Sp7-Taz-GFP

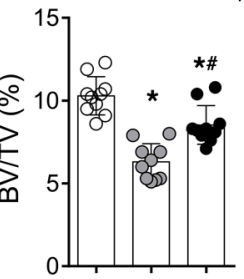

B

WT +
rAAV9-Sp7

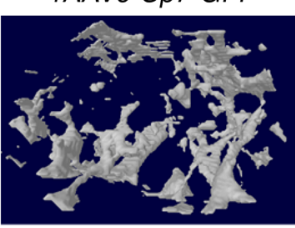

Bmncr-KO + rAAV9-Sp7-GFP

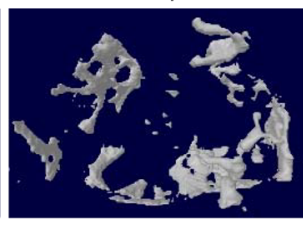

Bmncr-KO + rAAV9-Sp7-Taz-GFP

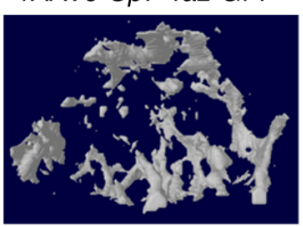

C

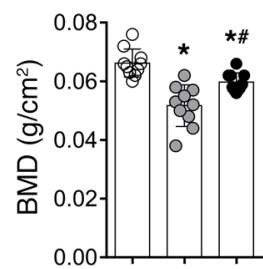

WT + rAAV9-Sp7-GFP

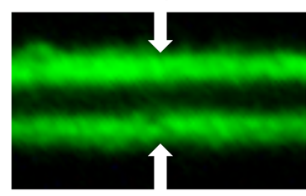
rAAV9-Sp7-GFP

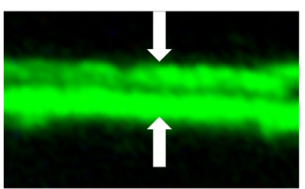
rAAV9-Sp7-Taz-GFP

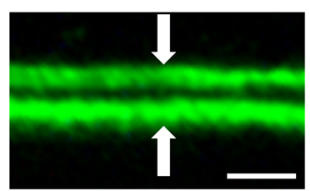

$\mathbf{F}$

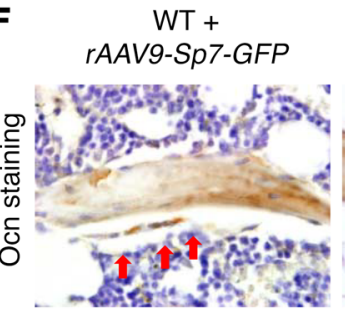

WT +

H

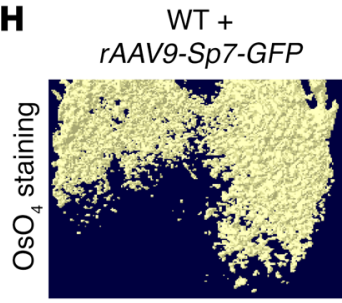

Bmncr-KO + rAAV9-Sp7-GFP

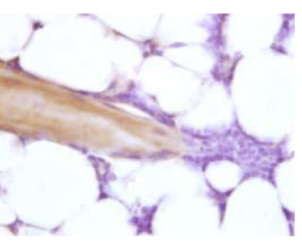

Bmncr-KO + rAAV9-Sp7-GFP

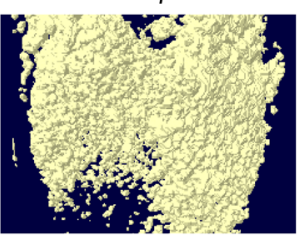

Bmncr-KO+ rAAV9-Sp7-Taz-GFP

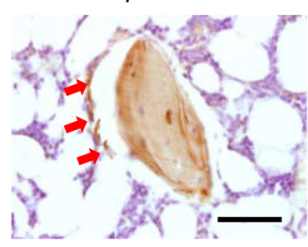

Bmncr-KO+ rAAV9-Sp7-Taz-GFP

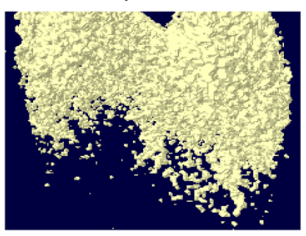

E

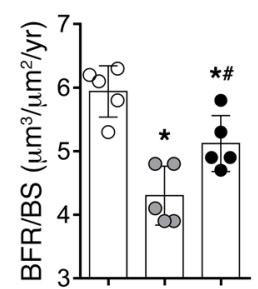

G

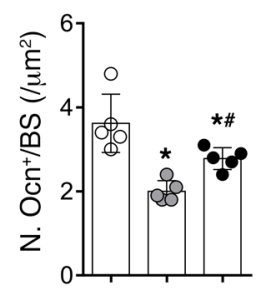

Figure 9. Overexpression of TAZ partially rescued the premature bone aging of Bmncr-KO mice. (A) BMD in femurs from 9-month-old WT and BmncrKO mice treated with rAAV9-Sp7-Taz-GFP or rAAV9-Sp7- GFP for 3 months were measured by dual-energy x-ray absorptiometry scan. (B-C) Representative micro-CT images and quantitative micro-CT analysis of trabecular bone mass in femurs ( $n=10$ per group). ( $\mathbf{D}-\mathbf{E})$ Calcein double-labeling-based quantification of bone formation rate per bone surface (BFR/BS) in femurs ( $n=5$ per group). White arrows in $\mathbf{D}$ show the distance between the 2 calcein labeling lines. Scale bar: $25 \mu \mathrm{M}$. Representative images of osteocalcin immunohistochemical staining (F) and quantification of number of osteoblasts (C). Red arrows in F represent osteocalcin-positive-staining cells. (H-J) OsO staining of decalcified femurs by micro-CT analysis and number and volume of adipocytes in distal femurs. Scale bar: $100 \mu \mathrm{M} . n=5$ per group. Data are mean \pm SD. ${ }^{*} P<0.05$ versus WT+rAAV9-Sp7-GFP group; ${ }^{*} P<0.05$ versus Bmncr-KO+rAAV9-Sp7-GFP group (1-way ANOVA).

of Bmncr-KO mice. We utilized rAAV serotype 9 with $S p 7$ promoter for gene delivery of Taz (rAAV9-Sp7-Taz-GFP) to osteoprogenitors in Bmncr-KO mice by intraosseous injection. Taz expression in $\mathrm{SP}^{+}$ osteoprogenitors was roughly 15 times higher in the mice infected with rAAV9-Sp7-Taz-GFP (Supplemental Figure 17A) than in control animals. rAAV9-Sp7-Taz-GFP-infected cells in bone marrow were further characterized by in situ immunofluorescence staining after injection. Immunofluorescence staining using SP7 and GFP antibodies demonstrated that most $\mathrm{GFP}^{+}$cells were $\mathrm{SP}^{+}$, indicating the successful construction and high efficiency and specificity of rAAV9-Sp7-Taz-GFP (Supplemental Figure 17B). We found that overexpression of $\mathrm{Taz}$ in osteoprogenitors partially reversed the switch between osteoblasts and adipocytes in Bmncr-KO mice. Bmncr-KO mice with rAAV9-Sp7-Taz-GFP injections showed an increase in bone mass (Figure 9, A-C and Supplemental Figure 18, $\mathrm{A}$ and B), BFR (Figure 9, D and E) and osteoblast numbers (Figure 9, $F$ and $G$ ), and showed a reduced number and volume of adipocytes (Figure 9, H-J) compared with rAAV9-Sp7-GFP control. However, rAAV9-Sp7-Taz-GFP administration could not rescue the bone phenotype of Bmncr-KO mice to the basal level of WT control, suggesting that $\mathrm{Taz}$ overexpression can only partially compensate the Bmncr deficiency since Bmncr regulates both osteogenic niche and TAZ activities through the assembly of ABL and TAZ.

Restoring BMNCR level in human BMSCs reversed age-related switch between osteoblast and adipocyte differentiation. Given that Bmncr regulates the lineage fate of murine BMSCs, we tested whether human BMNCR (LOC107985249) could promote osteogenic differentiation and inhibit adipogenic differentiation of human 
A
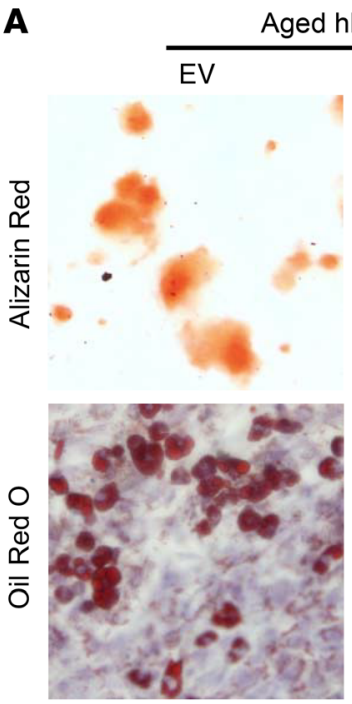
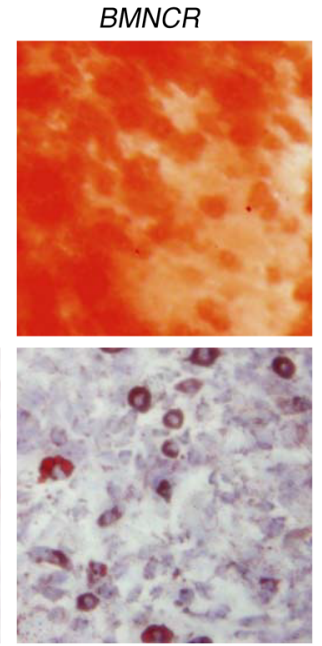

B

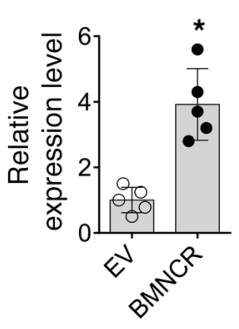

D

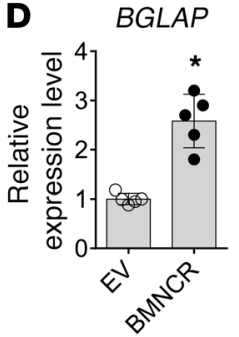

C $\quad A L P L$

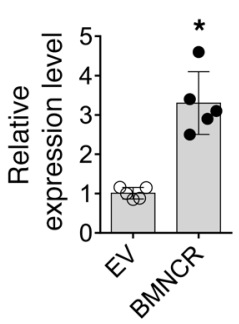

E
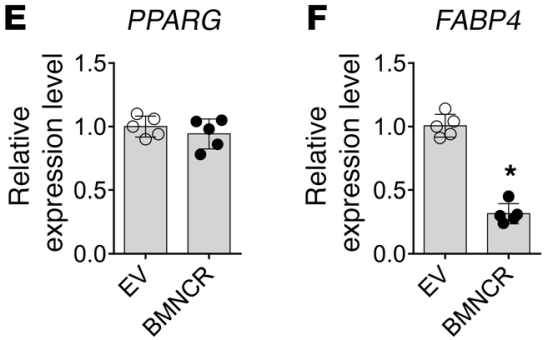

G
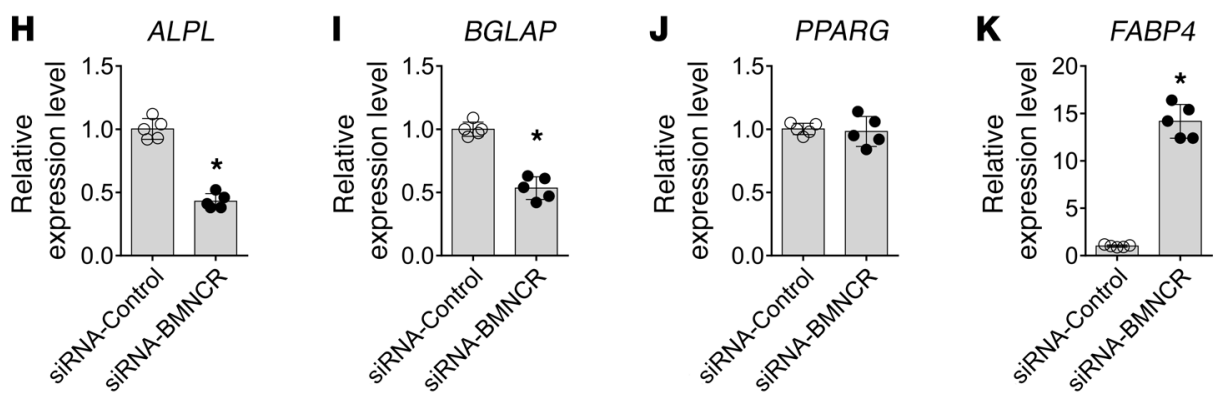

Figure 10. BMNCR promoted osteogenic differentiation and inhibits adipogenic differentiation of human BMSCs. (A) Representative images of Alizarin Red $S$ staining (original magnification $\times 200$ ) and Oil Red 0 staining (original magnification $\times 1,000$ ) in aged human BMSCs transfected with $B M N C R$ plasmid or empty vector (EV) and followed with osteogenic induction or adipogenic induction, respectively. Data are representative of 3 independent experiments. (B-F) qRT-PCR analysis of the levels of SP7, ALPL, BGLAP, PPARG, and FABP4 expression. $n=5$ per group. Data are mean \pm SD. ${ }^{*} P<0.05$ (Student's $t$ test). (G-K) qRT-PCR analysis of the levels of SP7, ALPL, BCLAP, PPARG, and FABP4 expression in young human BMSCs transfected with SiRNA-BMNCR or siRNA-control and followed with osteogenic induction or adipogenic induction, respectively. $n=5$ per group. Data are mean \pm SD. ${ }^{*} P<0.05$ (Student's $t$ test).

BMSCs. Human BMSCs isolated from aged patients (ages ranging from 65-73 years) were subjected to transfection of BMNCR plasmid and followed with osteogenic differentiation and adipogenic differentiation induction, respectively. Aged human BMSCs with BMNCR overexpression showed increased osteogenic differentiation ability as detected by Alizarin Red staining and qRT-PCR analysis with $S P 7, A L P L$, and BGLAP primers (Figure 10, A-D). Overexpression of $B M N C R$ inhibited the FABP4 expression and lipid formation in aged human BMSCs with adipogenic differentiation induction (Figure 10, A, E, and F). Human BMSCs isolated from young patients (ages ranging from 20-31 years) with transfection with siRNA-BMNCR showed reduced osteogenic differentiation ability and enhanced adipogenic differentiation compared with the siRNA-control group (Figure 10, G-K). These data indicate that BMNCR restoration is capable of reversing the age-related lineage shift between osteoblast and adipocyte differentiation in human BMSCs.

\section{Discussion}

Age-related lineage switching of BMSCs between osteogenic and adipogenic fates is critical in age-associated osteoporosis $(5,6)$. In the present study, we identified a lncRNA, Bmncr, that orchestrates osteogenic or adipogenic commitment of BMSCs during aging. $B m n c r$ regulated the osteogenic niche of BMSCs through the modulation of extracellular matrix protein, fibromodulin, and activation of the BMP2 pathway. Bmncr also served as a scaffold for the TAZ and ABL complex to promote the assembly of TAZ with RUNX2/ PPARG, which enhances osteogenesis and inhibits adipogenesis. Elevation of Bmncr levels in mouse and human BMSCs reversed age-related cell fate switches between osteoblasts and adipocytes. In this manner, Bmncr plays a key role in the age-related osteogenic niche alteration and lineage fate shift of BMSCs.

Bmncr deficiency in mice resulted in premature bone aging, which is characterized by reduced bone formation and increased bone marrow fat accumulation. Since the depletion of Bmncr in the Bmncr-KO mouse model is not restricted to BMSCs, we cannot exclude the potential involvement of Bmncr ablation in other tissues. We did perform Bmncr tissue expression pattern analysis and showed that Bmncr levels were high in bone tissue and white fat tissue, but low in lung, brain, muscle, kidney, and other tissues. Yet we did not observe changes in body weight, suggesting that Bmncr 
may have little effect on fat and other tissue. We also measured the expression level of Bmncr in BMSCs, osteoblasts, monocytes, and preosteoclasts in bone marrow. Compared with BMSCs and osteoblasts, the expression of Bmncr in monocytes and preosteoclasts was much lower. In addition, bone resorption in Bmncr-KO mice was not affected. Our in vitro assessment showed that BMSCs transfected with siRNA-Bmncr inclined to differentiate into adipocytes rather than osteoblasts, implying direct effects of Bmncr on BMSC lineage fate. Moreover, transgenic mice with specific expression of Bmncr in LepR-expressing cells, which are the main source of osteoblasts and adipocytes in bone marrow, showed increased bone mass and reduced marrow fat, suggesting that Bmncr has direct effects on bone through regulating lineage fate of BMSCs.

Factors that might impact Bmncr expression are rather interesting to study. We discovered that oxidative stress regulated Bmncr expression in BMSCs; however, there are possibilities that other factors may also affect Bmncr expression. During aging, senescent cells secrete certain factors and change the surrounding environment. In 2008, Coppé et al. first quantitatively analyzed the factors secreted by senescent cells and proposed the concept of a senescence-associated secretory phenotype (SASP) consisting of cytokines, chemokines, growth factors, and proteases (31). A recent study reports that the clearance of senescent cells prevents age-related bone loss (32). Whether Bmncr expression in BMSCs is controlled by the SASP of surrounding senescent cells in bone marrow is worthy of further study.

The stem cell niche is defined as a specialized microenvironment composed of cells, cytokines, and the extracellular matrix (ECM) that houses stem cells and maintains the balance of selfrenewal, quiescence, and lineage fate commitment. Several types of cells have been identified as essential hematopoietic stem cell niche components (33-35). Other than the cells identified as niche components, ECM has been shown to participate in determining the stem cell pools and regulating the shift in stem cell lineage fate (36). For example, ECM proteins biglycan and FMOD are 2 critical components that organize the niche of tendon stem/progenitor cells (21). The CTGF/VEGF complex in ECM modulates lineage fate of MSCs in arterial repair/remodeling after injury (37). In the present study, we found that Bmncr regulates an array of ECM proteins, including FMOD. Depletion of Bmncr leads to alteration of ECM composition and BMSC pool size in the trabecular-rich metaphysis region and central bone marrow. However, the mechanisms underlying the regulation of FMOD on cell adherence is not characterized in this study. Whether FMOD in the matrix could directly bind to the receptors on BMSC surfaces and therefore anchor the BMSCs to the matrix requires more study.

In this study, although we observed a decreased trend of $\mathrm{BMD}$, bone mass, and osteoblast number in Fmod-KO mice, the changes were not significant. Ablation of Fmod in Bmncr-Tg mice, however, significantly impaired the bone formation and enhanced marrow fat accumulation. These inconsistent data may be due to the amplified effects of Fmod in the situation of Bmncr overexpression. Yet, the absence of Fmod did not change the phenotype of Bmncr-Tg mice to the level of the WT mice, suggesting that in addition to FMOD, Bmncr also regulates bone mass through other signaling pathways. Therefore, we speculate that FMOD, as an ECM protein, is important for BMSC adherence to the metaphyseal area but may have marginal effects on osteogenic differentiation of BMSCs.

Cytokines are important components of the cell niche that function through activation or suppression of diverse signaling pathways (34). BMP2 belongs to the BMP family and is a wellknown potent inducer of osteogenic commitment of MSCs (25). We found BMP2 expression and active pSMAD1/5 levels were reduced in BMSCs after Bmncr knockdown. A previous study reported that TAZ/YAP transcriptional activity in endothelial cells regulates BMP expression during intramembranous ossification (38). In this study, we found that Bmncr promoted TAZ transcriptional activity via assembling TAZ and ABL. Thus, the decreased BMP2 expression in Bmncr-KO BMSCs may be a result of downregulated TAZ transcriptional activity.

TAZ, as a transcriptional coactivator for RUNX2-induced Bglap gene expression, is an important endogenous regulator of osteoblast differentiation $(29,39,40)$. TAZ has also been shown to suppress adipocyte differentiation by transcriptionally repressing PPARG-mediated gene expression $(29,30)$. A recent study reports that polycystins and TAZ integrate at the molecular level to reciprocally regulate osteoblast and adipocyte differentiation (40). We found that Bmncr served as a scaffold to assembly of TAZ and ABL and activation of the TAZ and RUNX2 transcriptional complex. Analyzing the bone phenotype of TAZ-null mice and crossing the TAZ-null mice with the Bmncr-KO mice to determine if there are additive decrements in bone mass would be an ideal technique to test the role of TAZ in Bmncr effects on BMSC lineage fate switch. Yet, the study cited earlier reported that TAZ-null mice showed severe dysfunction of the kidney, which may also contribute to the bone phenotype $(41,42)$. Therefore, we overexpressed Taz in osteoprogenitors in Bmncr-KO mice to see whether it has rescue effects. Bmncr-KO mice with rAAV-9-SP7-Taz-GFP injection showed an increased bone mass, elevated osteoblasts, and reduced adipocyte number compared with the control. rAAV-9-SP7-Taz-GFP administration, however, couldn't rescue the bone phenotype of Bmncr$K O$ mice to the basal level of WT controls, suggesting that Taz overexpression can only partially compensate the Bmncr deficiency since Bmncr regulates both osteogenic niche and TAZ activities through assembling ABL and TAZ.

lncRNAs have been reported to regulate gene expression patterns, binding of key transcription factors to promoters, and the physical association of transcription factors themselves (10, 14, 43). We found that Bmncr regulated Fmod transcription by modulating Fmod 3D chromatin structure. The effects of Bmncr on the other chromatin modification markers, such as histone modification and transcription factor binding on Fmod, will need further study. We found that mouse Bmncr has $75 \%$ sequence homology with human BMNCR. Further functional analysis of human $B M N C R$ showed that it also promoted osteoblastic differentiation and inhibited the adipogenic differentiation of BMSCs. These data suggest that human BMNCR also plays a key role in determining the cell fate shift of human BMSCs and age-related bone loss. The underlying mechanism of BMNCR function in human BMSCs is not characterized in this study.

The development of potential drugs to treat senile osteoporosis through targeting Bmncr and TAZ would be interesting. The discovery of compounds that stimulate Bmncr or TAZ expression 
in BMSCs is an attractive topic for future study. In the study by Xiao (40), a compound termed Zinc01442821 was discovered to promote TAZ signaling through regulating PC1/TAZ interactions. Another interesting strategy is to deliver exogenous Bmncr or TAZ specifically into BMSCs using a rAAV delivery system. The advantages of rAAV, such as low immunogenicity, lack of pathogenicity, and episomal persistence of the transgene, have led to rAAV being widely used in an increasing number of recent clinical trials. In this study, we found Bmncr-KO mice administrated with rAAV9-SP7-Taz-GFP showed an increased bone mass, elevated osteoblasts, and reduced marrow fat accumulation compared with rAAV9-SP7-GFP control. Thus, Bmncr and TAZ are promising targets for the treatment of senile osteoporosis.

In conclusion, our study identified an lncRNA, Bmncr, decreased during aging, that mediated age-related bone loss. We revealed a mechanistic strategy whereby lncRNA regulated BMSC lineage fate switching by modulating the osteogenic niche and interaction of TAZ and ABL. Our study may provide a new approach for the treatment of age-associated osteoporosis.

\section{Methods}

Animal models. We obtained the Bmncr-KO mice using the TetraOne technique from Cyagen Biosciences. The $4.92 \mathrm{~kb}$ sequence of Bmncr was selected as the knockout region. Mouse genomic fragments were amplified from the BAC clone with high-fidelity Taq DNA polymerase and were assembled into a targeting vector together with recombination sites and selection markers. Targeting vector was injected into the eggs of C57BL/6 (B6) mice. For genotyping of Bmncr, genomic DNA was extracted from tail tips.

We obtained LepR ${ }^{+}$cell-specific Bmncr transgenic $\left(\right.$Bmncr- $_{\text {LepR }}{ }^{T g}$ ) mice from Cyagen Biosciences. Briefly, LepR-Bmncr vector was constructed by subcloning the mouse Bmncr cDNA (synthesized by Cyagen Biosciences) into the SalI-EcoRI site in a plasmid containing the LepR promoter. The LepR-Bmncr plasmid was then transfected into BMSCs using Lipofectamine 2000 (Invitrogen). qRT-PCR was used to detect the expression of Bmncr compared with an empty vector control. The LepR-Bmncr fragment was then purified and microinjected into C57BL/6J F2 mouse oocytes, and the oocytes were surgically transferred into pseudopregnant C57BL/6J dams. Two lines with higher levels of Bmncr expression were selected from 6 transgenic founders and bred in C57BL/ 6 strain for 6 generations to obtain offspring with a defined genetic background. One line with 10 -fold overexpression of $B m n c r$ was used for studies. The WT mice were used as controls.

We obtained the Fmod-null mice (Fmod-KO) by CRISPR/Cas9-mediated genome engineering from Cyagen Biosciences. Exon 2 was selected as the target site. Cas 9 and gRNA were coinjected into fertilized eggs for KO mouse production. The pups were genotyped by PCR analysis.

All mice were maintained in a C57BL/6 background. LepR-Cre mice (catalog 008320) and Rosa26-EYFP $\mathrm{P}^{\ell / f l}$ mice (catalog 006148) were purchased from Jackson Laboratory. The male mice were used for experiments.

BMSC isolation and culture. Mouse BMSCs were isolated as previously described (5). Briefly, tibiae and femurs of mice were collected and crushed with a mortar and pestle. The bone was then digested with collagenase A (MilliporeSigma) to obtain a single-cell suspension. Cells within the supernatant were collected and incubated with phycoerythrin-conjugated (PE-conjugated), FITC-conjugated, peridi- nin chlorophyll protein-conjugated (PerCP-conjugated), and allophycocyanin-conjugated (APC-conjugated) antibodies that recognized mouse Sca-1 (BioLegend, 108108), CD29 (BioLegend, 102206), CD45 (BioLegend, 103132), and CD11b (BioLegend, 101226) for 20 minutes at $4^{\circ} \mathrm{C}$. For human BMSCs, human bone marrow cells were collected and incubated with FITC-, APC-, and PE-conjugated antibodies that recognized human Stro-1 (BioLegend, 340106), CD45 (BioLegend, 304012), and CD146 (BioLegend, 361008) at $4^{\circ} \mathrm{C}$ for 30 minutes. The acquisition was performed on a FACS Aria model (BD Biosciences), and the analysis was performed using FACS DIVE software version 6.1.3 (BD Biosciences).

The sorted mouse Sca- $1^{+} \mathrm{CD} 29^{+} \mathrm{CD} 45^{-} \mathrm{CD}_{11 b^{-}}$BMSCs and human CD $146{ }^{+}$Stro- $1^{+}$CD $45^{-}$BMSCs were cultured to reach $80 \%-85 \%$ confluence. Then, first-passage BMSCs were detached and seeded in culture flasks for the enrichment of cell populations. Only third-passage BMSCs were subjected to induction of adipogenic and osteogenic differentiation.

Adipogenic differentiation assay. For adipogenic differentiation of BMSCs in vitro, BMSCs with transfection of siRNA-Bmncr (RiboBio Co.) or Bmncr-plasmid (Sangon Biotech) were cultured in 6-well plates at $2.5 \times 10^{6}$ cells/well with $\alpha$-MEM containing $10 \%$ fetal bovine serum, $0.5 \mathrm{mM}$ 3-isobutyl-1-methylxanthine, $5 \mu \mathrm{g} / \mathrm{ml}$ insulin, and $1 \mu \mathrm{M}$ dexamethasone for 10 days. Culture medium was changed every other day. Oil Red O (Sigma-Aldrich) staining was performed to detect lipid in mature adipocytes.

Osteogenic differentiation assay. For osteoblastic differentiation, BMSCs with transfection of siRNA-Bmncr (RiboBio Co.) or Bmncrplasmid (Sangon Biotech) were cultured in 24-well plates at $5 \times 10^{5}$ cells/well with $\alpha$-MEM containing $10 \%$ fetal bovine serum, $0.1 \mathrm{mM}$ dexamethasone, $10 \mathrm{mM}$ b-glycerol phosphate, and $50 \mathrm{mM}$ ascorbate-2-phosphate for 21 days (44). Then, cells were stained with $2 \%$ Alizarin Red S (Sigma-Aldrich) at pH 4.2 to evaluate the cell matrix mineralization. A Diaphot Inverted Microscope and Camera System (Nikon) was used for imaging. Alizarin Red S released from the cell matrix into the cetyl-pyridinium chloride solution was quantified by spectrophotometry at $540 \mathrm{~nm}$.

IncRNA microarray assay. IncRNA microarray assay was performed by the Clinical Microarray Core at the University of California, Los Angeles (UCLA). Briefly, total RNA was extracted from BMSCs isolated from 3-month-old and 18-month-old female C57BL/6 mice, and then labeled with $\mathrm{Cy} 3$. The fragmentation mixtures were hybridized to an Arraystar Mouse Long Noncoding RNA Microarray (Agilent). Feature Extraction Software 10.7.1.1 (Agilent) analyzed the scanned images using default parameters to obtain background subtracted and spatially detrended processed signal intensities as the raw data. Raw data were normalized in a quantile algorithm with Genespring 12.0 (Agilent). Probes for which at least $100 \%$ of samples in any 1 condition out of 2 conditions that had flags in "Detected" were maintained. The raw data have been uploaded to GEO with the series record GSE 116925 (https://www.st-va.ncbi.nlm.nih.gov/geo/query/ acc.cgi acc $=$ GSE116925).

GeneChip microarray assay. The GeneChip microarray assay was performed by Oebiotech Company. RNAs were extracted from WT and Bmncr-KO-derived BMSCs. The fragmentation mixtures were hybridized to an Affymetrix mouse 1.0 gene ST array. Robust multiarray average was used to perform the data normalization. Principal component analysis in Partek Genomics Suite was used to perform the cluster analysis of different samples. ANOVA was used to identify 
the differential expression genes. The raw data have been uploaded to GEO with the series record GSE116926 (https://www.st-va.ncbi.nlm. nih.gov/geo/query/acc.cgi?acc=GSE116926).

Histochemistry analysis. Histochemistry analysis was performed as previously described (45). Briefly, femurs were harvested from mice after euthanasia, fixed in $10 \%$ formalin for 24 hours, decalcified in 10\% EDTA for 14-21 days, and embedded in paraffin. Bone sections (4- $\mu \mathrm{m}$-thick, longitudinally oriented) were stained with $\mathrm{H} \& \mathrm{E}$ and TRAP (both from Sigma-Aldrich) to quantify the number and surface of osteoblasts, number and surface of osteoclasts, and number and area of adipocytes.

Histomorphometric analysis of 2D parameters of the trabecular bones in femur was performed using OsteoMeasureXP Software (OsteoMetrics, Inc.). The sample area selected for histomorphometric analysis was a $1 \mathrm{~mm}^{2}$ area within the metaphyseal secondary spongiosa, originating $1 \mathrm{~mm}$ below the growth plate. Osteoblast surface per bone surface (Ob.S/BS) and osteoblast number per bone perimeter (N.Ob/B.Pm) parameters were used to measure the bone formation. The parameters measured for bone resorption were osteoclast surface per bone surface (Oc.S/BS) and osteoclast number per bone perimeter (N.Oc/B.Pm).

Immunohistochemical and immunofluorescence staining. Immunohistochemical staining was performed as previously described (46). Briefly, bone sections were processed for antigen retrieval by digestion with $0.05 \%$ trypsin at $37^{\circ} \mathrm{C}$ for 15 minutes, and then incubated with primary antibody against osteocalcin (Takara, M173) overnight at $4^{\circ} \mathrm{C}$. Subsequently, an HRP-streptavidin detection system (Dako) was used to detect the immunoactivity, followed by counterstaining with hematoxylin (MilliporeSigma). Sections incubated with polyclonal rabbit IgG (R\&D Systems) served as negative controls.

For immunofluorescence staining, we incubated the sections with primary antibodies against leptin receptor (R\&D Systems, BAF497), osteocalcin (Takara, M173), SP7 (Abcam, ab22552), perilipin (Abcam, ab172907), and GFP (Rockland, 600-101-215). Nuclei were counterstained with DAPI (Sigma). The sections were mounted with the ProLong Antifade Kit (Molecular Probes) and observed under a Fluo View 300 Confocal Microscope (Olympus America) and confocal microscope (FLUOVIEW FV300, Olympus).

qRT-PCR analysis. qRT-PCR analysis was performed using a Roche Molecular Light Cycler (Roche) as previously described (47). Total RNA from cells was isolated using Trizol reagent (Invitrogen). Reverse transcription was performed using $1 \mu \mathrm{g}$ total RNA and SuperScript II (Invitrogen). Amplification reactions were set up in $25 \mu \mathrm{l}$ reaction volumes containing SYBR Green PCR Master Mix (PE Applied Biosystems), $1 \mu \mathrm{l}$ volume of cDNA, and amplification primers. Primer sequences are listed in Supplemental Table 1 and Supplemental Table 2.

Immunoprecipitation and Western blot analysis. Immunoprecipitation was performed as previously described (37). Briefly, total cell lysates were collected and immunoprecipitated by incubation with the ABL antibodies (Santa Cruz, sc-56887), followed by adsorption to protein $\mathrm{G}$ sepharose. Immunoprecipitates were separated by SDS-PAGE and blotted onto a PVDF membrane (Bio-Rad Laboratories). The membrane was incubated with antibodies against TAZ (Cell Signaling Technology, 4883S) and visualized by enhanced chemiluminescence (ECL Kit, Amersham Biosciences).

For Western blot analysis, total cell lysates were separated by SDS-PAGE and blotted on PVDF membranes (Millipore). The membranes were incubated with specific antibodies against FMOD (Abcam, ab81443), pSMAD1/5 (Novus Biologicals, SI17-06), SMAD1/5 (Novus Biologicals, SY09-03), RUNX2 (Abcam, ab23981), SP7 (Abcam, ab22552), PPARG (Cell Signaling Technology, 2443), Fabp4 (Abcam, ab13979), TAZ (Cell Signaling Technology, 4883), ABL (Santa Cruz, sc-56887), and $\beta$-actin (Proteintech, 60008-1-Ig), then reprobed with appropriate HRP-conjugated secondary antibodies. Blots were visualized by enhanced chemiluminescence (ECL Kit, Amersham Biosciences). The complete unedited blots are shown in Supplemental Materials.

Micro-CT analysis. The right femurs were dissected from mice and fixed with $4 \%$ paraformaldehyde for 24 hours, then scanned and analyzed with high-resolution micro-CT (Skyscan 1172, Bruker MicroCT). We used NRecon image reconstruction software version 1.6 (Bruker MicroCT), CTAn data analysis software version 1.9 (Bruker MicroCT), and CTVol 3D model visualization software version 2.0 (Bruker MicroCT) to analyze parameters of the trabecular bone in the metaphysis and cortical bone in the mid-diaphysis. The scanner was set at 50 $\mathrm{kVp}, 201 \mu \mathrm{A}$, and a resolution of $12.64 \mathrm{~mm} /$ pixel. For the distal femur, the region of interest selected for analysis was $5 \%$ of the femoral length from $0.1 \mathrm{~mm}$ below the growth plate to determine trabecular bone volume per tissue volume (Tb.BV/TV), trabecular number (Tb.N), trabecular separation (Tb.Sp), and trabecular thickness (Tb.Th).

$\mathrm{OsO}_{4}$ staining and micro-CT analysis. Whole intact femurs were dissected from mice, fixed in phosphate-buffered paraformaldehyde for 48 hours, and decalcified for 2 weeks in 10\% EDTA. Then the femurs and tibiae were stained in $2 \%$ aqueous osmium tetroxide $\left(\mathrm{OsO}_{4}\right.$, Sigma-Aldrich) for 2 hours. The bones were rinsed in water for 48 hours and then scanned at $6 \mu \mathrm{m}$ resolution using $45 \mathrm{kVp}$ and $177 \mu \mathrm{A}$ (Skyscan 1172, Bruker MicroCT). Quantification of fat volume, density, and distribution throughout the marrow was registered to lowcontrast decalcified bone as previously described $(48,49)$.

RNA immunoprecipitation and RNA pull-down. RNA immunoprecipitation was performed using a Magna RIP RNA-Binding Protein Immunoprecipitation Kit (17-700, Millipore) according to the manufacturer's instructions. RNA for in vitro experiments was transcribed using a T7 High Yield RNA Synthesis Kit (E2040S, New England Biolabs) according to the manufacturer's instructions. The 3 '-end biotin-labeled RNA probes used in RNA pull-down were generated using an RNA 30 End Biotinylation Kit (20160, Pierce) according to the manufacturer's instructions. Each pull-down assay used $5 \mathrm{pM}$ of 30-biotinylated RNA.

Chromatin conformation capture. $3 \mathrm{C}$ experiments were performed essentially as described $(50,51)$. In brief, $3 \times 10^{6}$ to $5 \times 10^{6}$ formaldehyde-fixed BMSCs were transduced with control or targeting siRNABMNCR and lysed with lysis buffer (10 mM Tris- $\mathrm{HCl} \mathrm{pH} \mathrm{8,} 10 \mathrm{mM}$ $\mathrm{NaCl}, 0.3 \%$ IGEPAL-CA-630, 0.3\% Triton X-100, $1 \mathrm{X}$ protease inhibitor). Nuclei were digested with DpnII and ligated with T4 DNA ligase. Primers were designed to amplify indicated ligated regions, and were quantified by real-time PCR. Values were normalized to an intronic region of the Fmod gene that does not contain a DpnII cut site.

Virus preparation and injection. Recombinant adeno-associated serotype 9 virus with Sp7 promoter for gene delivery of TAZ system (rAAV9-Sp7-TAZ-GFP) was generated by OBIO Technology. Both TAZ and GFP were cloned into the rAAV9 vector under the control of Sp7 promotor. Clones were confirmed by DNA sequencing prior to use and virus titers were determined by dot blot. AAV viral titer of $5 \times 10^{12} \mathrm{vec}-$ tor genomes/ml were used in the study. RAAV9-Sp7-GFP was used as control. Mice received $5 \mu \mathrm{l}$ of either rAAV9-SP7-TAZ-GFP or rAAV9- 
SP7-GFP via periosteal injection into medullary cavity of femur twice per month for 3 months.

Study population. Human bone marrow samples were obtained from 18 patients with osteoarthritis undergoing knee joint replacement ( 8 male and 10 female, age range $42-72$ years); 16 patients with femoral neck fracture and/or femoral head fractures undergoing hip joint replacement ( 7 male and 9 female, age range $44-73$ years); and 14 patients with tibia fractures ( 8 male and 6 female, age range 20-62 years) and 16 patients with femur shaft fracture ( 9 male and 5 female, age range 20-62 years) undergoing open reduction internal fixation. Human bone marrow aspiration and collection were conducted by the Orthopedic Surgery Department at Xiangya Hospital of Central South University. A total of 64 patients (32 male and 32 female) were selected on the basis of the inclusion and exclusion criteria. All subjects were screened using a detailed questionnaire, disease history, and physical examination. Subjects were excluded from the study if they had conditions affecting bone metabolism, including diseases of the kidney, liver, parathyroid, thyroid, diabetes mellitus, hyperprolactinemia, oophorectomy, rheumatoid arthritis, ankylosing spondylitis, malabsorption syndromes, malignant tumors, hematologic diseases, or previous pathological fractures within 1 year. If the subjects had received treatment with glucocorticoids, estrogens, thyroid hormone, parathyroid hormone, fluoride, bisphosphonate, calcitonin, thiazide diuretics, barbiturates, or antiseizure medication, they were also excluded.

Statistics. Data are mean \pm SD. For comparisons of 2 groups, a 2-tailed Student's $t$ test was used. Comparisons of multiple groups were made using a 1- or 2-way ANOVA. All experiments were repeated at least 3 times, and representative experiments are shown. Differences were considered significant at $P$ less than 0.05 .

Study approval. The clinical study was approved by the Ethics Committee of Xiangya Hospital of Central South University. All animal care protocols and experiments were reviewed and approved by the Animal Care and Use Committees of the Laboratory Animal Research Center at Xiangya Medical School of Central South University. All mice were maintained in the specific pathogen-free facility of the Laboratory Animal Research Center at Central South University. Written informed consent was obtained from all participants prior to bone marrow aspiration and collection.

\section{Author contributions}

XHL, CJL, and YX designed the experiments. CJL, YX, and MY carried out most of the experiments. QG, TS, XS, and YH helped to collect the samples. XHL and CJL supervised the experiments, analyzed results, and wrote the manuscript.

\section{Acknowledgments}

We acknowledge Papergoing Co. for help editing and proofreading the English in the final manuscript. This work was supported by the Major International (Regional) Joint Research Project of China National Natural Scientific Foundation (NSFC) (grant 81520108008), the Training Program of the Major Research Plan of NSFC (grant 91749105), the National Natural Science Foundation of China (grants 81570806, 81700785, 81873669, 81802209), the Hunan Province Natural Science Foundation of China (grant 2018JJ3863), the Innovation Driven Project of Central South University (grant 20170033010017), and the Talent Plan of Xiangya Hospital in Central South University (grants 35 and 41).

Address correspondence to: Xiang-Hang Luo, Department of Endocrinology, Endocrinology Research Center, Xiangya Hospital of Central South University, 87 Xiangya Road, Changsha, Hunan 410008, China. Phone: 86.731.4328888; Email: xianghangluo @hotmail.com.
1. Pittenger MF, et al. Multilineage potential of adult human mesenchymal stem cells. Science. 1999;284(5411):143-147.

2. Discher DE, Mooney DJ, Zandstra PW. Growth factors, matrices, and forces combine and control stem cells. Science. 2009;324(5935):1673-1677.

3. Guilak F, Cohen DM, Estes BT, Gimble JM, Liedtke W, Chen CS. Control of stem cell fate by physical interactions with the extracellular matrix. Cell Stem Cell. 2009;5(1):17-26.

4. Moerman EJ, Teng K, Lipschitz DA, LeckaCzernik B. Aging activates adipogenic and suppresses osteogenic programs in mesenchymal marrow stroma/stem cells: the role of PPAR-gamma2 transcription factor and TGF-beta/BMP signaling pathways. Aging Cell. 2004;3(6):379-389.

5. Li CJ, et al. MicroRNA-188 regulates age-related switch between osteoblast and adipocyte differentiation. J Clin Invest. 2015;125(4):1509-1522.

6. Li H, et al. FOXP1 controls mesenchymal stem cell commitment and senescence during skeletal aging. J Clin Invest. 2017;127(4):1241-1253.

7. Klattenhoff CA, et al. Braveheart, a long noncoding RNA required for cardiovascular lineage commitment. Cell. 2013;152(3):570-583.

8. Yang L, et al. ncRNA- and Pc2 methylationdependent gene relocation between nuclear structures mediates gene activation programs. Cell. 2011;147(4):773-788.

9. Ng SY, Johnson R, Stanton LW. Human long non-coding RNAs promote pluripotency and neuronal differentiation by association with chromatin modifiers and transcription factors. EMBO J. 2012;31(3):522-533.

10. Sun L, et al. Long noncoding RNAs regulate adipogenesis. Proc Natl Acad Sci U S A. 2013;110(9):3387-3392.

11. Guttman M, et al. lincRNAs act in the circuitry controlling pluripotency and differentiation. Nature. 2011;477(7364):295-300.

12. Guttman M, et al. Chromatin signature reveals over a thousand highly conserved large non-coding RNAs in mammals. Nature. 2009;458(7235):223-227.

13. Guttman M, et al. Ab initio reconstruction of cell type-specific transcriptomes in mouse reveals the conserved multi-exonic structure of lincRNAs. Nat Biotechnol. 2010;28(5):503-510.

14. Tsai MC, et al. Long noncoding RNA as modular scaffold of histone modification complexes. Science. 2010;329(5992):689-693.

15. Tang Y, et al. TGF-beta1-induced migration of bone mesenchymal stem cells couples bone resorption with formation. Nat Med. 2009;15(7):757-765.

16. Morán I, et al. Human $\beta$ cell transcriptome analysis uncovers lncRNAs that are tissue-specific, dynamically regulated, and abnormally expressed in type 2 diabetes. Cell Metab. 2012;16(4):435-448.

17. Marrelli M, Paduano F, Tatullo M. Cells isolated from human periapical cysts express mesenchymal stem cell-like properties. Int J Biol Sci. 2013;9(10):1070-1078.

18. Siclari VA, et al. Mesenchymal progenitors residing close to the bone surface are functionally distinct from those in the central bone marrow. Bone. 2013;53(2):575-586.

19. Zhou BO, Yue R, Murphy MM, Peyer JG, Morrison SJ. Leptin-receptor-expressing mesenchymal stromal cells represent the main source of bone formed by adult bone marrow. Cell Stem Cell. 2014;15(2):154-168.

20. Kalamajski S, Oldberg A. Fibromodulin binds collagen type I via Glu-353 and Lys-355 in leucine-rich repeat $11 . J$ Biol Chem. 2007;282(37):26740-26745.

21. Bi Y, et al. Identification of tendon stem/progenitor cells and the role of the extracellular matrix in their niche. Nat Med. 2007;13(10):1219-1227.

22. Sjöberg A, Onnerfjord P, Mörgelin M, Heinegård D, Blom AM. The extracellular matrix and inflammation: fibromodulin activates the classical pathway of complement by directly binding C1q. J Biol Chem. 2005;280(37):32301-32308. 
23. Cong L, et al. Multiplex genome engineering using CRISPR/Cas systems. Science. 2013;339(6121):819-823.

24. Mali P, et al. RNA-guided human genome engineering via Cas9. Science. 2013;339(6121):823-826.

25. Wang EA, et al. Recombinant human bone morphogenetic protein induces bone formation. Proc Natl Acad Sci U S A. 1990;87(6):2220-2224.

26. Chaudhari A, Ron E, Rethman MP. Recombinant human bone morphogenetic protein-2 stimulates differentiation in primary cultures of fetal rat calvarial osteoblasts. Mol Cell Biochem. 1997;167(1-2):31-39.

27. Gori F, Schipani E, Demay MB. Fibromodulin is expressed by both chondrocytes and osteoblasts during fetal bone development. J Cell Biochem. 2001;82(1):46-57.

28. Yue R, Zhou BO, Shimada IS, Zhao Z, Morrison $S$ J. Leptin receptor promotes adipogenesis and reduces osteogenesis by regulating mesenchymal stromal cells in adult bone marrow. Cell Stem Cell. 2016;18(6):782-796.

29. Hong JH, et al. TAZ, a transcriptional modulator of mesenchymal stem cell differentiation. Science. 2005;309(5737):1074-1078.

30. Matsumoto Y, et al. Reciprocal stabilization of ABL and TAZ regulates osteoblastogenesis through transcription factor RUNX2. J Clin Invest. 2016;126(12):4482-4496.

31. Coppé JP, et al. Senescence-associated secretory phenotypes reveal cell-nonautonomous functions of oncogenic RAS and the p53 tumor suppressor. PLoS Biol. 2008;6(12):2853-2868.

32. Farr JN, et al. Targeting cellular senescence prevents age-related bone loss in mice. Nat Med.
2017;23(9):1072-1079.

33. Sugiyama T, Kohara H, Noda M, Nagasawa T. Maintenance of the hematopoietic stem cell pool by CXCL12-CXCR4 chemokine signaling in bone marrow stromal cell niches. Immunity. 2006;25(6):977-988.

34. Morrison SJ, Spradling AC. Stem cells and niches: mechanisms that promote stem cell maintenance throughout life. Cell. 2008;132(4):598-611.

35. Li L, Clevers H. Coexistence of quiescent and active adult stem cells in mammals. Science. 2010;327(5965):542-545.

36. Engler AJ, Sen S, Sweeney HL, Discher DE. Matrix elasticity directs stem cell lineage specification. Cell. 2006;126(4):677-689.

37. Li C, et al. RhoA determines lineage fate of mesenchymal stem cells by modulating CTGF-VEGF complex in extracellular matrix. Nat Commun. 2016;7:11455.

38. Uemura M, Nagasawa A, Terai K. Yap/Taz transcriptional activity in endothelial cells promotes intramembranous ossification via the BMP pathway. Sci Rep. 2016;6:27473.

39. Byun MR, et al. Canonical Wnt signalling activates TAZ through PP1A during osteogenic differentiation. Cell Death Differ. 2014;21(6):854-863.

40. Xiao Z, et al. Polycystin-1 interacts with TAZ to stimulate osteoblastogenesis and inhibit adipogenesis. J Clin Invest. 2018;128(1):157-174.

41. Makita R, et al. Multiple renal cysts, urinary concentration defects, and pulmonary emphysematous changes in mice lacking TAZ. Am J Physiol Renal Physiol. 2008;294(3):F542-F553.

42. Hossain Z, et al. Glomerulocystic kidney disease in mice with a targeted inactivation of Wwtr1.
Proc Natl Acad Sci U S A. 2007;104(5):1631-1636.

43. Aguilo F, Zhou MM, Walsh MJ. Long noncoding RNA, polycomb, and the ghosts haunting INK4b-ARF-INK4a expression. Cancer Res. 2011;71(16):5365-5369.

44. Li H, et al. A novel microRNA targeting HDAC5 regulates osteoblast differentiation in mice and contributes to primary osteoporosis in humans. JClin Invest. 2009;119(12):3666-3677.

45. Li C, et al. Disruption of LRP6 in osteoblasts blunts the bone anabolic activity of PTH. J Bone Miner Res. 2013;28(10):2094-2108.

46. Li C, Williams BO, Cao X, Wan M. LRP6 in mesenchymal stem cells is required for bone formation during bone growth and bone remodeling. Bone Res. 2014;2:14006.

47. Xie H, et al. PDGF-BB secreted by preosteoclasts induces angiogenesis during coupling with osteogenesis. Nat Med. 2014;20(11):1270-1278.

48. Xiao Z, Zhang S, Cao L, Qiu N, David V, Quarles LD. Conditional disruption of Pkd1 in osteoblasts results in osteopenia due to direct impairment of bone formation. J Biol Chem. 2010;285(2):1177-1187.

49. Xiao Z, et al. Osteoblast-specific deletion of $\mathrm{Pkd} 2$ leads to low-turnover osteopenia and reduced bone marrow adiposity. PLOS ONE. 2014;9(12):e114198.

50. Pasquali L, et al. Pancreatic islet enhancer clusters enriched in type 2 diabetes risk-associated variants. Nat Genet. 2014;46(2):136-143.

51 . Tena JJ, et al. An evolutionarily conserved three-dimensional structure in the vertebrate Irx clusters facilitates enhancer sharing and coregulation. Nat Commun. 2011;2:310. 